\title{
Bifurcation Analysis of a Transversely Cracked Nonlinear Jeffcott Rotor System at Different Resonance Cases
}

\author{
Nasser A. Saeed and Mostafa Eissa \\ Department of Physics and Engineering Mathematics, Faculty of Electronic Engineering, Menouf, 32952, \\ Menoufia University, Egypt.
}

(Received 12 November 2016; accepted 26 April 2017)

This work focuses on the dynamical behaviour and bifurcations of a vertically supported Jeffcott rotor system having a transverse crack and nonlinear stiffness characteristics at the primary, sub-harmonic, and super-harmonic resonance cases. The nonlinear restoring force due to the bearing-clearance, the crack breathing, the disc eccentricity, and the orientation angle between the crack and imbalance direction are considered in the system model. The equations governing the system motion are derived and solved analytically by applying the Multiple Scales Perturbation Technique (MSPT). The slow-flow modulating equations are obtained and the spinning speed response curve is plotted. The whirling orbit and amplitude spectrum are constructed in the three considered resonance cases. The acquired results provide a better understanding of the main reasons of the super- and sub-harmonic resonance excitations. In additions, we concluded that the suitable resonance case that can be used for early detections of the cracks in the rotating shafts is the sub-harmonic resonance case. Finally, the obtained results are confirmed numerically and compared with the work published in the literature.

\section{NOMENCLATURE}

$y_{1}, \dot{y}_{1}, \ddot{y}_{1}$
$y_{2}, \dot{y}_{2}, \ddot{y}_{2}$
$a_{1}, a_{2}$
$\mu_{1}, \mu_{2}$
$\omega$
$\beta$
$\Omega$
$\gamma$
$f$
$\alpha$

Dimensionless displacement, velocity, and acceleration of the disc geometric center at $Y_{1}$-direction

Dimensionless displacement, velocity, and acceleration of the disc geometric center at $Y_{2}$-direction

Dimensionless whirling amplitudes of the disc geometric center at $Y_{1}$ and $Y_{2}$-directions. Dimensionless linear damping coefficients at $Y_{1}$ and $Y_{2}$-directions, respectively.

Dimensionless linear natural frequency of the cracked system.

Dimensionless cubic nonlinearity coefficient.

Dimensionless spinning speed of the disc.

The orientation angle between the crack direction and imbalance direction.

Dimensionless disc-eccentricity magnitude.

Dimensionless parameter representing the relative reduction of the shaft-stiffness due to the crack.

\section{INTRODUCTION}

Rotating machines have an important role in modern industry due to their numerous applications such as automobile engines, turbo-machinery, pumps, high-speed compressors, generators, aerospace, steam and gas turbines, etc. The existence of vibration in such machines is an inherent phenomenon, which initiates because of the dynamic interaction between the stator and rotating parts, the shaft cracks, and the mass imbalance. The existence of cracks in a machine shaft can eventually lead to catastrophic failures and dangerous accidents. There- fore, studying the dynamic behaviour of cracked rotors has received considerable attention of researchers for many years.

Comprehensive reviews on the dynamic behaviour of a rotor system with transverse cracks have been presented by Wauer ${ }^{1}$ and Dimarogonas, ${ }^{2}$ where the authors reported many dynamical phenomena that can be used to diagnose the presence of cracks on machine rotors. Gasch ${ }^{3}$ investigated the dynamic behaviours of a simple rotor system with a transverse crack on its shaft by using the hinge model. Based on the breathing crack model, the equations of motion for a simple rotor system has been formulated and studied by Jun, et al. ${ }^{4}$ The authors reported that the vibration behaviour due to the shaft crack could be recognized from the second horizontal harmonic components measured near the second harmonic resonant speed. Cheng, et al. ${ }^{5}$ discussed the influences of the orientation angle between the crack direction and the imbalance eccentricity on the vibrations level of a cracked Jeffcott rotor system at synchronous whirling. They concluded that the maximum vibration peak occurs when the orientation angle is zero, while the minimum vibration peak happens at orientation angle equal to $\pi$.

Sinuou and Lees ${ }^{6}$ applied the alternate frequency/time domain approach to analyze the dynamic response of a rotor system having a breathing crack. It was found that the change of the dynamic characteristics of the rotor system near half of the resonant speed is a positive indication of the presence of a breathing crack. Sinuou and Lees ${ }^{7,8}$ introduced nonlinear studies to the rotor system with a cracked shaft. The harmonic balance and the continuation method have been combined to analyze the system periodic motion. The authors concluded that the whirling motion at half the critical speed and at the critical speed could be considered as indicators of the existence of a breathing crack. In addition, Sinuou ${ }^{9}$ studied a cracked rotor system numerically with the crack breathing model. He 
illustrated that the evolutions of the sub-harmonic resonance component that was excited at 2:1 and 3:1 resonance cases can be used to detect the cracks in the rotating machines.

Chen, et al. ${ }^{10}$ discussed the influence of the crack depth on the whirling amplitude of a rotor system. The authors reported that, at the critical speed, the whirling amplitude does not affect the crack depth if it is less than $50 \%$. However, the small crack depth has a significant effect on the whirling motion at higher harmonics. Han and $\mathrm{Chu}^{11}$ investigated the effects of double cracks on the dynamic behaviour of the rotorbearing system. The authors concluded that the existence of two cracks on the rotor shaft have a significant influence on system behaviour that differs completely from that of a system with one crack. Dai and Chen ${ }^{12}$ investigated the nonlinear response of a cracked rotor system that had asymmetrical viscoelastic supports. The authors utilized the harmonic balance method to study the effect of different parameters such as the crack-depth, crack-position, disc-position and disc-thickness. Lin and $\mathrm{Chu}^{13}$ investigated the effect of torsional excitation on a cracked rotor system. AL-Shudeifat, et al. ${ }^{14}$ applied harmonic balance method and breathing crack mechanism to explore the dynamic characteristics of a cracked rotor system. They concluded that the results from the theoretical model are very close to the practical system if the length and diameter of the shaft are large. However, when the crack thickness is narrow, the breathing of the crack becomes more similar to the theoretical model. Jun and Gadala ${ }^{15,16}$ analyzed the dynamic behaviour of the cracked rotor system using the additional slope and bending moment at the crack position. Ishida ${ }^{17}$ studied different resonant cases of cracked Jeffcott rotor system. Hou, et al. ${ }^{18}$ investigated both 2:1 and 3:1 sub-harmonic resonances of aircraft cracked rotor systems that were subjected to maneuver load. The authors applied the multiple scales perturbation method to study local bifurcations of the system at the considered resonance cases.

In most articles concerning crack detection in rotating machines, the authors used the linear model of a horizontally supported Jeffcott rotor system to simulate the dynamic behaviour change due to existence of a crack on their shafts. The most applied analytical techniques are the numerical ones. Additionally, the dynamic behaviour of a vertically supported Jeffcott rotor system with nonlinear stiffness characteristics has not been investigated. Therefore, a vertically supported Jeffcott rotor system with nonlinear stiffness and a transverse crack on its shaft has been studied within this article. The disc eccentricity, the breathing crack mechanism, and the orientation angle between the crack and imbalance directions are considered in the system model. Multiple scales perturbation technique is employed to obtain an analytical solution at the different resonance cases. Bifurcation analysis for the different system parameters is conducted. Then, the acquired analytical results are confirmed numerically. The presented results may contribute to better understanding of the main reasons of the super- and sub-harmonic resonance excitations, and the orientation angle that has a significant effect on the oscillation amplitude. The suitable resonance case that can be used for early detections of a crack in the rotating shafts has been defined. By the end, a comparison with previously published work is included.

\section{SYSTEM MODELLING AND APPROXIMATE SOLUTION}

Figures $1 \mathrm{a}$ and $1 \mathrm{~b}$ illustrates a schematic diagram for a vertically supported Jeffcott rotor system, where $G$ denotes the disc geometric center, $O$ is the intersection point of both the disc plane (i.e. $Y_{1} Y_{2}$ plane) and the bearing axis $\left(O Y_{3}\right)$, and $r$ represents the horizontal distance between $G$ and $O$. The restoring force $F_{r}$ by the shaft stiffness and the supporting bearings is considered a nonlinear function of $r$ such that $F_{r}=k_{1} r+k_{2} r^{3}$. Accordingly, the restoring forces at $Y_{1}$ and $Y_{2}$ directions are $F_{Y_{1}}=F_{r} \cos (\theta t)=k_{1} y_{1}+k_{2}\left(y_{1}^{2}+y_{2}^{2}\right) y_{1}$ and $F_{Y_{2}}=F_{r} \sin (\theta t)=k_{1} y_{2}+k_{2}\left(y_{1}^{2}+y_{2}^{2}\right) y_{2}$. Therefore, the equations that govern the dynamical behaviours of a vertically supported Jeffcott rotor system that having a transverse-crack on its shaft can be expressed as follows: $:^{18-20}$

$$
\begin{aligned}
m \ddot{y}_{1}+c_{1} \dot{y}_{1}+k_{1} y_{1}+ & k_{2}\left(y_{1}^{3}+y_{1} y_{2}^{2}\right)= \\
& m e_{d} \theta^{2} \cos (\theta t+\gamma)+R_{1} ; \\
m \ddot{y}_{2}+c_{2} \dot{y}_{2}+k_{1} y_{2}+ & k_{2}\left(y_{2}^{3}+y_{1}^{2} y_{2}\right)= \\
& m e_{d} \theta^{2} \sin (\theta t+\gamma)+R_{2} ;
\end{aligned}
$$

where $m$ is the mass of the disc, $c_{1}$ and $c_{2}$ are the linear damping coefficients, $k_{1}$ and $k_{2}$ are the linear and nonlinear stiffness coefficients, $e_{d}$ represents the disc eccentricity, $\theta$ is the disc spinning speed, $\gamma$ is the orientation angle between the crack direction $(G \zeta)$ and the imbalance direction $(G e), R_{1}, R_{2}$ represent the parametric forces exerted due to the time varying stiffness that resulting from the shaft crack, $O-Y_{1} Y_{2} Y_{3}$ is stationary-coordinate system, $O-y_{1} y_{2}$ is the loci of the geometric-center $G$, and $O-\zeta \eta$ is a rotational coordinate system, which rotates by the disc spinning speed.

\subsection{Parametric Forces Due to the Crack- Breathing}

It is assumed that the initial tendency of the rotational axis $O \zeta$ (i.e. the crack direction) is parallel to $Y_{1}$-axis as illustrated in Fig. 1c. Therefore, the parametric forces at $Y_{1}$ and $Y_{2}$ directions are $R_{1}$ and $R_{2}$, respectively, which can be represented as follows: ${ }^{3,5,18}$

$$
\begin{aligned}
R_{1} & =F(\theta t) \Delta k y_{\zeta} \cos (\theta t) \\
& =F(\theta t) \Delta k\left[y_{1} \cos (\theta t)+y_{2} \sin (\theta t)\right] \cos (\theta t) \\
R_{2} & =F(\theta t) \Delta k y_{\zeta} \sin (\theta t) \\
& =F(\theta t) \Delta k\left[y_{1} \cos (\theta t)+y_{2} \sin (\theta t)\right] \sin (\theta t)
\end{aligned}
$$

where $\Delta k$ represents the maximum reduction of the shaft stiffness when the crack is fully opened, and $\Delta k F(\theta t)$ describes the variation of the shaft-stiffness as a function of the rotation angle $(\theta t)$, where $F(\theta t)$ is given as follows:

$$
F(\theta t)=\frac{1}{2}(1+\cos (\theta t))
$$

The derivation of Eqs. (2a), (2b) and (3) can be found in detail in Gasch. ${ }^{3}$ During the rotation of the shaft, the crack opens and closes according to rotational angle magnitude as shown in Fig. 2, where if the rotational angle $\theta t \approx 0^{\circ}$, the crack is considered fully opened, while at $\theta t \approx 180^{\circ}$, the crack is assumed fully closed. Substituting Eq. (3) into Eqs. (2a)-(2b), 


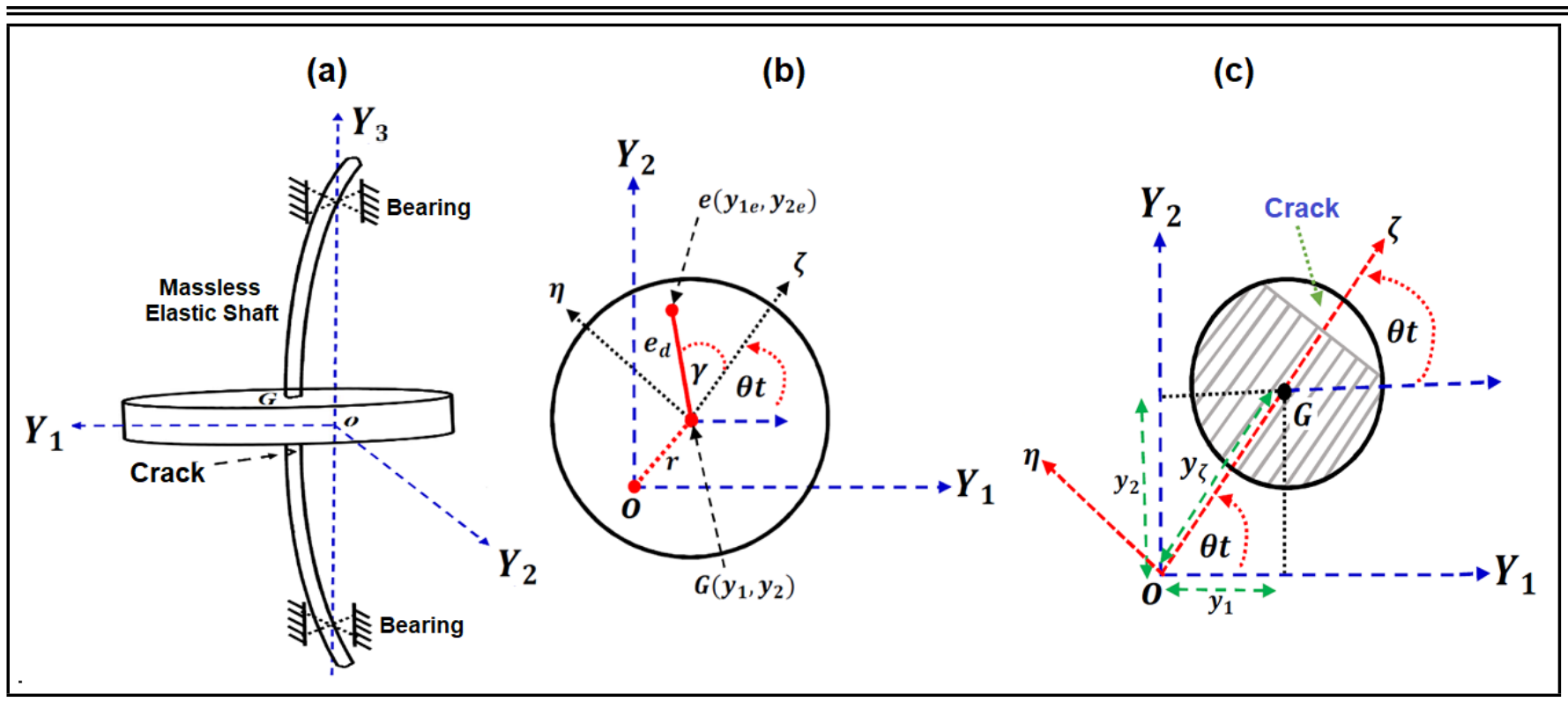

Figure 1. (a) Jeffcott rotor system, (b) coordinate system of the disc, and (c) Schematic diagram of the cracked shaft cross section.

and then inserting the resulting equations into Eqs. (1a)-(1b), we get

$$
\begin{gathered}
m \ddot{y}_{1}+c_{1} \dot{y}_{1}+\left(k_{1}-\frac{\Delta k}{4}\right) y_{1}+k_{2} y_{1}^{3}+k_{2} y_{1} y_{2}^{2}= \\
m e_{d} \theta^{2} \cos (\theta t+\gamma)+ \\
\frac{\Delta k}{4}\left[y_{1} \sum_{j=1}^{3} q_{j} \cos (j \theta t)+y_{2} \sum_{j=1}^{3} f_{j} \sin (j \theta t)\right] ; \\
m \ddot{y}_{2}+c_{2} \dot{y}_{2}+\left(k_{1}-\frac{\Delta k}{4}\right) y_{2}+k_{2} y_{2}^{3}+k_{2} y_{1}^{2} y_{2}= \\
m e_{d} \theta^{2} \sin (\theta t+\gamma)+ \\
\frac{\Delta k}{4}\left[y_{2} \sum_{j=1}^{3} p_{j} \cos (j \theta t)+y_{1} \sum_{j=1}^{3} f_{j} \sin (j \theta t)\right] .
\end{gathered}
$$

Introducing the dimensionless parameters $t^{*}=\sqrt{\frac{k_{1}}{m}} t, y_{1}^{*}=$ $\frac{y_{1}}{e}, y_{2}^{*}=\frac{y_{2}}{e}$ into Eqs. (4a)-(4b), and omitting the asterisk for brevity (where $e$ is a representative length that has a magnitude of the same order as the imbalance $e_{d}$ ), we get the following dimensionless equations of motion

$$
\begin{aligned}
& \ddot{y}_{1}+ \mu_{1} \dot{y}_{1}+\omega^{2} y_{1}+\beta y_{1}^{3}+\beta y_{1} y_{2}^{2}= \\
& f \Omega^{2} \cos (\Omega t+\gamma)+ \\
& \alpha\left[y_{1} \sum_{j=1}^{3} q_{j} \cos (j \Omega t)+y_{2} \sum_{j=1}^{3} f_{j} \sin (j \Omega t)\right] ; \\
& \ddot{y}_{2}+\mu_{2} \dot{y}_{2}+\omega^{2} y_{2}+\beta y_{2}^{3}+\beta y_{2} y_{1}^{2}= \\
& f \Omega^{2} \sin (\Omega t+\gamma)+ \\
& \alpha\left[y_{1} \sum_{j=1}^{3} f_{j} \sin (j \Omega t)+y_{2} \sum_{j=1}^{3} p_{j} \cos (j \Omega t)\right] ;
\end{aligned}
$$

where $\mu_{1}=\frac{c_{1}}{\sqrt{k_{1} m}}, \mu_{2}=\frac{c_{2}}{\sqrt{k_{1} m}}, \alpha=\frac{\Delta k}{4 k_{1}}, \beta=\frac{e^{2} k_{2}}{k_{1}}$, $\omega=\sqrt{1-\alpha}, \Omega=\theta \sqrt{\frac{m}{k_{1}}}, f=\frac{e_{d}}{e}, q_{1}=\frac{3}{2}, q_{2}=f_{2}=1$, $q_{3}=f_{1}=f_{3}=p_{1}=\frac{1}{2}, p_{2}=-1, p_{3}=\frac{-1}{2}$.

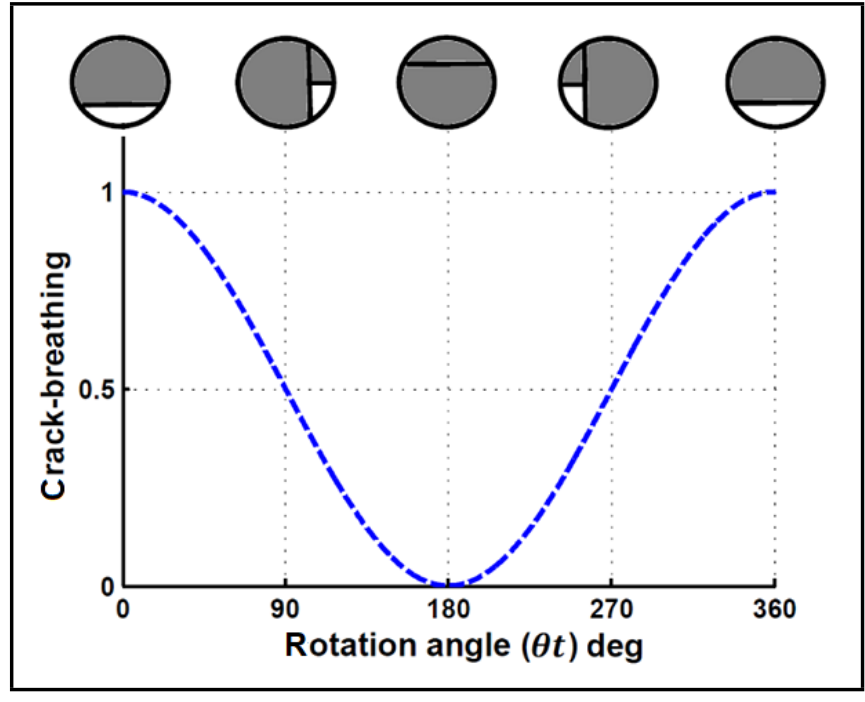

Figure 2. Model of stiffness-variation for the breathing crack.

\subsection{Perturbation Analysis}

Applying MSPT ${ }^{21-23}$ an approximate solution can be obtained to Eqs. (5a)- $(5 b)$ by seeking the solution in the following form:

$$
y_{k}(t, \varepsilon)=y_{k 1}\left(\tau_{0}, \tau_{1}\right)+\varepsilon y_{k 2}\left(\tau_{0}, \tau_{1}\right)+O\left(\varepsilon^{2}\right) \text { and } k=1,2 ;
$$

where $\varepsilon$ is a book-keeping dimensionless perturbation parameter, $\tau_{0}=t$ and $\tau_{1}=\varepsilon t$ are the fast and slow time scales. In terms of $\tau_{0}$ and $\tau_{1}$, the time derivatives can be written as

$$
\begin{aligned}
\frac{d}{d t} & =D_{0}+\varepsilon D_{1} ; & \frac{d^{2}}{d t^{2}} & =D_{0}^{2}+2 \varepsilon D_{0} D_{1} ; \\
D_{j} & =\frac{\partial}{\partial \tau_{j}} ; & j & =0,1 .
\end{aligned}
$$

Parameters of Eqs. (5a)-(5b) had to be scaled so that the system damping, nonlinearities, and excitation forces appeared in the same perturbed equation as

$$
\mu_{1}=\varepsilon \mu_{1} ; \quad \mu_{2}=\varepsilon \mu_{2} ; \quad \alpha=\varepsilon \alpha ; \quad \beta=\varepsilon \beta ; \quad f=\varepsilon f .
$$


Substituting Eqs. (6)-(8) into Eqs. (5a)-(5b), and equating coefficients of the same powers of $\varepsilon$, we get the following:

$O\left(\varepsilon^{0}\right)$ :

$$
\begin{aligned}
& \left(D_{0}^{2}+\omega^{2}\right) y_{11}=0 \\
& \left(D_{0}^{2}+\omega^{2}\right) y_{21}=0
\end{aligned}
$$

$O\left(\varepsilon^{1}\right):$

$$
\begin{aligned}
& \left(D_{0}^{2}+\omega^{2}\right) y_{12}=-2 D_{0} D_{1} y_{11}-\mu_{1} D_{0} y_{11}-\beta y_{11}^{3}- \\
& \beta y_{11} y_{21}^{2}+f \Omega^{2} \cos \left(\Omega \tau_{0}+\gamma\right)+ \\
& \quad \frac{1}{2} \alpha y_{11}\left[3 \cos \left(\Omega \tau_{0}\right)+2 \cos \left(2 \Omega \tau_{0}\right)+\cos \left(3 \Omega \tau_{0}\right)\right]+ \\
& \quad \frac{1}{2} \alpha y_{21}\left[\sin \left(\Omega \tau_{0}\right)+2 \sin \left(2 \Omega \tau_{0}\right)+\sin \left(3 \Omega \tau_{0}\right)\right] ; \quad(10 \mathrm{a}) \\
& \left(D_{0}^{2}+\omega^{2}\right) y_{22}=-2 D_{0} D_{1} y_{21}-\mu_{2} D_{0} y_{21}-\beta y_{21} y_{11}^{2}- \\
& \quad \beta y_{21}^{3}+f \Omega^{2} \sin \left(\Omega \tau_{0}+\gamma\right)+ \\
& \quad \frac{1}{2} \alpha y_{11}\left[\sin \left(\Omega \tau_{0}\right)+2 \sin \left(2 \Omega \tau_{0}\right)+\sin \left(3 \Omega \tau_{0}\right)\right]+ \\
& \frac{1}{2} \alpha y_{21}\left[\cos \left(\Omega \tau_{0}\right)-2 \cos \left(2 \Omega \tau_{0}\right)-\cos \left(3 \Omega \tau_{0}\right)\right] . \quad(10 \mathrm{~b})
\end{aligned}
$$

The solution of Eqs. (9a)-(9b) can be formulated as

$$
\begin{aligned}
& y_{11}=Q_{1}\left(\tau_{1}\right) e^{i \omega \tau_{0}}+\bar{Q}_{1}\left(\tau_{1}\right) e^{-i \omega \tau_{0}} \\
& y_{21}=Q_{2}\left(\tau_{1}\right) e^{i \omega \tau_{0}}+\bar{Q}_{2}\left(\tau_{1}\right) e^{-i \omega \tau_{0}} .
\end{aligned}
$$

The coefficients $Q_{1}\left(\tau_{1}\right)$ and $Q_{2}\left(\tau_{1}\right)$ are unknown functions up to this step of analysis, and the over bar denotes the complex conjugate function. Inserting Eqs. (11a)-(11b) into Eqs. (10a)(10b), we get

$$
\begin{aligned}
& \left(D_{0}^{2}+\omega^{2}\right) y_{12}=-\left(2 i \omega D_{1} Q_{1}+\mu_{1} i \omega Q_{1}+3 \beta Q_{1}^{2} \bar{Q}_{1}+\right. \\
& \left.\beta \bar{Q}_{1} Q_{2}^{2}+2 \beta Q_{1} Q_{2} \bar{Q}_{2}\right) e^{i \omega \tau_{0}}-\beta\left(Q_{1}^{3}+Q_{1} Q_{2}^{2}\right) e^{3 i \omega \tau_{0}}+ \\
& \frac{f \Omega^{2}}{2} e^{i \gamma} e^{i \Omega \tau_{0}}+\frac{1}{4} \alpha\left(3 Q_{1}-i Q_{2}\right) e^{i(\Omega+\omega) \tau_{0}}+ \\
& \frac{1}{4} \alpha\left(3 \bar{Q}_{1}-i \bar{Q}_{2}\right) e^{i(\Omega-\omega) \tau_{0}}+\frac{1}{2} \alpha\left(Q_{1}-i Q_{2}\right) e^{i(2 \Omega+\omega) \tau_{0}}+ \\
& \frac{1}{2} \alpha\left(\bar{Q}_{1}-i \bar{Q}_{2}\right) e^{i(2 \Omega-\omega) \tau_{0}}+\frac{1}{4} \alpha\left(Q_{1}-i Q_{2}\right) e^{i(3 \Omega+\omega) \tau_{0}}+ \\
& \quad \frac{1}{4} \alpha\left(\bar{Q}_{1}-i \bar{Q}_{2}\right) e^{i(3 \Omega-\omega) \tau_{0}}+c c ; \\
& \left(D_{0}^{2}+\omega^{2}\right) y_{22}=-\left(2 i \omega D_{1} Q_{2}+\mu_{2} i \omega Q_{2}+3 \beta Q_{2}^{2} \bar{Q}_{2}+\right. \\
& \left.\beta Q_{1}^{2} \bar{Q}_{2}+2 \beta Q_{1} \bar{Q}_{1} Q_{2}\right) e^{i \omega \tau_{0}}-\beta\left(Q_{2}^{3}+Q_{1}^{2} Q_{2}\right) e^{3 i \omega \tau_{0}}- \\
& \frac{i f \Omega^{2}}{2} e^{i \gamma} e^{i \Omega \tau_{0}}+\frac{1}{4} \alpha\left(Q_{2}-i Q_{1}\right) e^{i(\Omega+\omega) \tau_{0}}+ \\
& \frac{1}{4} \alpha\left(\bar{Q}_{2}-i \bar{Q}_{1}\right) e^{i(\Omega-\omega) \tau_{0}}-\frac{1}{2} \alpha\left(Q_{2}+i Q_{1}\right) e^{i(2 \Omega+\omega) \tau_{0}}- \\
& \frac{1}{2} \alpha\left(\bar{Q}_{2}+i \bar{Q}_{1}\right) e^{i(2 \Omega-\omega) \tau_{0}}-\frac{1}{4} \alpha\left(Q_{2}+i Q_{1}\right) e^{i(3 \Omega+\omega) \tau_{0}}- \\
& \frac{1}{4} \alpha\left(\bar{Q}_{2}+i \bar{Q}_{1}\right) e^{i(3 \Omega-\omega) \tau_{0}}+c c ;
\end{aligned}
$$

where $c c$ denotes the complex conjugate of the preceding terms, The reported resonance cases from Eqs. (12a)-(12b) are the primary resonance $(\Omega \cong \omega)$, sub-harmonic resonance $(\Omega \cong 2 \omega)$, and super-harmonic resonance $\left(\Omega \cong \frac{2}{3} \omega\right)$ cases. The closeness of the spinning speed $(\Omega)$ to the obtained resonance cases can be described quantitatively via introducing the detuning parameters $\sigma_{1}, \sigma_{2}$, and $\sigma_{3}$ as follows:

$$
\begin{aligned}
& \Omega=\omega+\sigma_{1}=\omega+\varepsilon \hat{\sigma}_{1} ; \\
& \Omega=2 \omega+\sigma_{2}=2 \omega+\varepsilon \hat{\sigma}_{2} ; \\
& \Omega=\frac{2}{3} \omega+\sigma_{3}=\frac{2}{3} \omega+\varepsilon \hat{\sigma}_{3} .
\end{aligned}
$$

\subsubsection{Primary Resonance Case $\left(\Omega=\omega+\sigma_{1}\right)$}

Inserting the first relation in Eq. (13) into Eqs. (12a)-(12b), yields

$$
\begin{gathered}
\left(D_{0}^{2}+\omega^{2}\right) y_{12}=-\left(2 i \omega D_{1} Q_{1}+\mu_{1} i \omega Q_{1}+3 \beta Q_{1}^{2} \bar{Q}_{1}+\right. \\
\beta \bar{Q}_{1} Q_{2}^{2}+2 \beta Q_{1} Q_{2} \bar{Q}_{2}-\frac{f \Omega^{2}}{2} e^{i \gamma} e^{i \sigma_{1} \tau_{1}}- \\
\left.\frac{1}{2} \alpha\left(\bar{Q}_{1}-i \bar{Q}_{2}\right) e^{i 2 \sigma_{1} \tau_{1}}\right) e^{i \omega \tau_{0}}+N S T \\
\left(D_{0}^{2}+\omega^{2}\right) y_{22}=-\left(2 i \omega D_{1} Q_{2}+\mu_{2} i \omega Q_{2}+3 \beta Q_{2}^{2} \bar{Q}_{2}+\right. \\
\beta Q_{1}^{2} \bar{Q}_{2}+2 \beta Q_{1} \bar{Q}_{1} Q_{2}+\frac{i f \Omega^{2}}{2} e^{i \gamma} e^{i \sigma_{1} \tau_{1}}+ \\
\left.\frac{1}{2} \alpha\left(\bar{Q}_{2}+i \bar{Q}_{1}\right) e^{2 i \sigma_{1} \tau_{1}}\right) e^{i \omega \tau_{0}}+N S T
\end{gathered}
$$

where NST stands for non-secular terms. The solvability conditions of Eqs. (14a)-(14b) are

$$
\begin{gathered}
2 i \omega D_{1} Q_{1}+\mu_{1} i \omega Q_{1}+3 \beta Q_{1}^{2} \bar{Q}_{1}+\beta \bar{Q}_{1} Q_{2}^{2}+2 \beta Q_{1} Q_{2} \bar{Q}_{2}- \\
\frac{f \Omega^{2}}{2} e^{i \gamma} e^{i \sigma_{1} \tau_{1}}-\frac{1}{2} \alpha\left(\bar{Q}_{1}-i \bar{Q}_{2}\right) e^{i 2 \sigma_{1} \tau_{1}}=0 ; \\
2 i \omega D_{1} Q_{2}+\mu_{2} i \omega Q_{2}+3 \beta Q_{2}^{2} \bar{Q}_{2}+\beta Q_{1}^{2} \bar{Q}_{2}+2 \beta Q_{1} \bar{Q}_{1} Q_{2}+ \\
\frac{i f \Omega^{2}}{2} e^{i \gamma} e^{i \sigma_{1} \tau_{1}}+\frac{1}{2} \alpha\left(\bar{Q}_{2}+i \bar{Q}_{1}\right) e^{2 i \sigma_{1} \tau_{1}}=0 .
\end{gathered}
$$

To analyze Eqs. (15a)-(15b), we put $Q_{1}$ and $Q_{2}$ in the polar form as

$$
\begin{aligned}
& Q_{1}=\frac{a_{1}}{2} e^{i \psi_{1}} \Rightarrow \\
& D_{1} Q_{1}=\frac{d}{d \tau_{1}} Q_{1}=\frac{d}{\varepsilon d t} Q_{1}=\frac{\dot{a}_{1}}{2 \varepsilon} e^{i \psi_{1}}+i \frac{a_{1}}{2 \varepsilon} \dot{\psi}_{1} e^{i \psi_{1}} \\
& Q_{2}=\frac{a_{2}}{2} e^{i \psi_{2}} \Rightarrow \\
& D_{1} Q_{2}=\frac{d}{d \tau_{1}} Q_{2}=\frac{d}{\varepsilon d t} Q_{2}=\frac{\dot{a}_{2}}{2 \varepsilon} e^{i \psi_{2}}+i \frac{a_{2}}{2 \varepsilon} \dot{\psi}_{2} e^{i \psi_{2}}
\end{aligned}
$$

where $a_{j}, \psi_{j}(j=1,2)$, denote the oscillation amplitudes and phases of the two vibrating modes. Substituting Eqs. (16a)- 
(16b) into Eqs. (15a)-(15b), yields

$$
\begin{aligned}
i \omega \dot{a}_{1}- & \omega a_{1} \dot{\psi}_{1}+\frac{i}{2} \varepsilon \mu_{1} \omega a_{1}+\frac{3}{8} \varepsilon \beta a_{1}^{3}+ \\
& \frac{1}{8} \varepsilon \beta a_{1} a_{2}^{2} e^{-i\left(2 \psi_{1}-2 \psi_{2}\right)}+\frac{1}{4} \varepsilon \beta a_{1} a_{2}^{2}- \\
& \frac{1}{2} \varepsilon f \Omega^{2} e^{i \gamma} e^{i\left(\sigma_{1} \tau_{1}-\psi_{1}\right)}-\frac{1}{4} \varepsilon \alpha a_{1} e^{2 i\left(\sigma_{1} \tau_{1}-\psi_{1}\right)}+ \\
& \frac{i}{4} \varepsilon \alpha a_{2} e^{i\left(2 \sigma_{1} \tau_{1}-\psi_{1}-\psi_{2}\right)}=0 \\
i \omega \dot{a}_{2}- & \omega a_{2} \dot{\psi}_{2}+\frac{i}{2} \varepsilon \mu_{2} \omega a_{2}+\frac{3}{8} \varepsilon \beta a_{2}^{3}+ \\
& \frac{1}{8} \varepsilon \beta a_{1}^{2} a_{2} e^{i\left(2 \psi_{1}-2 \psi_{2}\right)}+\frac{1}{4} \varepsilon \beta a_{1}^{2} a_{2}+ \\
& \frac{i}{2} \varepsilon f \Omega^{2} e^{i \gamma} e^{i\left(\sigma_{1} \tau_{1}-\psi_{2}\right)}+\frac{1}{4} \varepsilon \alpha a_{2} e^{2 i\left(\sigma_{1} \tau_{1}-\psi_{2}\right)}+ \\
& \frac{i}{4} \varepsilon \alpha a_{1} e^{i\left(2 \sigma_{1} \tau_{1}-\psi_{1}-\psi_{2}\right)}=0 .
\end{aligned}
$$

Returning each scaled parameter in Eqs. (17a)-(17b) to its original form (i.e. $\hat{\mu}_{1}=\frac{\mu_{1}}{\varepsilon}, \hat{\mu}_{2}=\frac{\mu_{2}}{\varepsilon}, \hat{\alpha}=\frac{\alpha}{\varepsilon}, \hat{\beta}=\frac{\beta}{\varepsilon}$, $\hat{f}=\frac{f}{\varepsilon}, \hat{\sigma}_{1}=\frac{\sigma_{1}}{\varepsilon^{2}}, t=\frac{\tau_{1}}{\varepsilon}$ ), while assuming $\phi_{1}=\sigma_{1} t-\psi_{1}$, and $\phi_{2}=\sigma_{1} t-\psi_{2}$, we get

$$
\begin{aligned}
i \omega \dot{a}_{1}- & \omega a_{1} \dot{\psi}_{1}+\frac{i}{2} \mu_{1} \omega a_{1}+\frac{3}{8} \beta a_{1}^{3}+\frac{1}{8} \beta a_{1} a_{2}^{2} e^{-i\left(2 \phi_{1}-2 \phi_{2}\right)}+ \\
& \frac{1}{4} \beta a_{1} a_{2}^{2}-\frac{1}{2} f \Omega^{2} e^{i\left(\phi_{1}+\gamma\right)}-\frac{1}{4} \alpha a_{1} e^{2 i \phi_{1}}+ \\
& \frac{i}{4} \alpha a_{2} e^{i\left(\phi_{1}+\phi_{2}\right)}=0
\end{aligned}
$$

$i \omega \dot{a}_{2}-\omega a_{2} \dot{\psi}_{2}+\frac{i}{2} \mu_{2} \omega a_{2}+\frac{3}{8} \beta a_{2}^{3}+\frac{1}{8} \beta a_{1}^{2} a_{2} e^{i\left(2 \phi_{2}-2 \phi_{1}\right)}+$

$$
\begin{aligned}
& \frac{1}{4} \beta a_{1}^{2} a_{2}+\frac{i}{2} f \Omega^{2} e^{i\left(\phi_{2}+\gamma\right)}+\frac{1}{4} \alpha a_{2} e^{2 i \phi_{2}}+ \\
& \frac{i}{4} \alpha a_{1} e^{i\left(\phi_{1}+\phi_{2}\right)}=0 .
\end{aligned}
$$

Eliminating $\psi_{1}, \psi_{2}$, and separating the real and imaginary parts of Eqs. (18a)-(18b), yields the following four autonomous slow-flow modulating equations that describe the evolution of both vibration amplitudes and their phases at the primary resonance

$$
\begin{aligned}
\dot{a}_{1}= & -\frac{1}{2} \mu_{1} a_{1}+\frac{1}{8 \omega} \beta a_{1} a_{2}^{2} \sin \left(2 \phi_{2}-2 \phi_{1}\right)+ \\
& \frac{1}{2 \omega} f \Omega^{2} \sin \left(\phi_{1}+\gamma\right)+\frac{1}{4 \omega} \alpha a_{1} \sin \left(2 \phi_{1}\right)- \\
& \frac{1}{4 \omega} \alpha a_{2} \cos \left(\phi_{1}+\phi_{2}\right) ; \\
\dot{a}_{2}= & -\frac{1}{2} \mu_{2} a_{2}-\frac{1}{8 \omega} \beta a_{1}^{2} a_{2} \sin \left(2 \phi_{2}-2 \phi_{1}\right)- \\
& \frac{1}{2 \omega} f \Omega^{2} \cos \left(\phi_{2}+\gamma\right)-\frac{1}{4 \omega} \alpha a_{2} \sin \left(2 \phi_{2}\right)- \\
& \frac{1}{4 \omega} \alpha a_{1} \cos \left(\phi_{1}+\phi_{2}\right) ;
\end{aligned}
$$

$$
\begin{aligned}
\dot{\phi}_{1}= & \sigma_{1}-\frac{3}{8 \omega} \beta a_{1}^{2}-\frac{1}{8 \omega} \beta a_{2}^{2} \cos \left(2 \phi_{2}-2 \phi_{1}\right)-\frac{1}{4 \omega} \beta a_{2}^{2}+ \\
& \frac{1}{2 a_{1} \omega} f \Omega^{2} \cos \left(\phi_{1}+\gamma\right)+\frac{1}{4 \omega} \alpha \cos \left(2 \phi_{1}\right)+ \\
& \frac{1}{4 a_{1} \omega} \alpha a_{2} \sin \left(\phi_{1}+\phi_{2}\right) ; \\
\dot{\phi}_{2}= & \sigma_{1}-\frac{3}{8 \omega} \beta a_{2}^{2}-\frac{1}{8 \omega} \beta a_{1}^{2} \cos \left(2 \phi_{2}-2 \phi_{1}\right)-\frac{1}{4 \omega} \beta a_{1}^{2}+ \\
& \frac{1}{2 a_{2} \omega} f \Omega^{2} \sin \left(\phi_{2}+\gamma\right)-\frac{1}{4 \omega} \alpha \cos \left(2 \phi_{2}\right)+ \\
& \frac{1}{4 a_{2} \omega} \alpha a_{1} \sin \left(\phi_{1}+\phi_{2}\right) .
\end{aligned}
$$

\subsubsection{Sub- and Super-Harmonic Resonance Cases}

$$
\left(\Omega=2 \omega+\sigma_{2}, \Omega=\frac{2}{3} \omega+\sigma_{3}\right)
$$

Substituting the second relation in Eq. (13) into Eqs. (12a)(12b), and following the same procedure applied in section 2.2.1, we can get the amplitude-phase modulating equations at sub-harmonic resonance case as

$$
\begin{aligned}
\dot{a}_{1}= & -\frac{1}{2} \mu_{1} a_{1}-\frac{1}{8 \omega} \beta a_{1} a_{2}^{2} \sin \left(\phi_{1}-\phi_{2}\right)+ \\
& \frac{3}{8 \omega} \alpha a_{1} \sin \left(\phi_{1}\right)-\frac{1}{8 \omega} \alpha a_{2} \cos \left(\frac{\phi_{1}+\phi_{2}}{2}\right) ; \\
\dot{a}_{2}= & -\frac{1}{2} \mu_{2} a_{2}-\frac{1}{8 \omega} \beta a_{1}^{2} a_{2} \sin \left(\phi_{2}-\phi_{1}\right)+ \\
& \frac{1}{8 \omega} \alpha a_{2} \sin \left(\phi_{2}\right)-\frac{1}{8 \omega} \alpha a_{1} \cos \left(\frac{\phi_{1}+\phi_{2}}{2}\right) ; \\
\dot{\phi}_{1}= & \sigma_{2}-\frac{3}{4 \omega} \beta a_{1}^{2}-\frac{1}{4 \omega} \beta a_{2}^{2} \cos \left(\phi_{1}-\phi_{2}\right)-\frac{1}{2 \omega} \beta a_{2}^{2}+ \\
& \frac{3}{4 \omega} \alpha \cos \left(\phi_{1}\right)+\frac{1}{4 \omega a_{1}} \alpha a_{2} \sin \left(\frac{\phi_{1}+\phi_{2}}{2}\right) ; \\
\dot{\phi}_{2}= & \sigma_{2}-\frac{3}{4 \omega} \beta a_{2}^{2}-\frac{1}{4 \omega} \beta a_{1}^{2} \cos \left(\phi_{2}-\phi_{1}\right)-\frac{1}{2 \omega} \beta a_{1}^{2}+ \\
& \frac{1}{4 \omega} \alpha \cos \left(\phi_{2}\right)+\frac{1}{4 \omega a_{2}} \alpha a_{1} \sin \left(\frac{\phi_{1}+\phi_{2}}{2}\right) ;
\end{aligned}
$$

where $\phi_{1}=\sigma_{2} t-2 \psi_{1}, \phi_{2}=\sigma_{2} t-2 \psi_{2}$.

From the third relation in Eq. (13) into Eqs. (12a)-(12b), we can get the following amplitude-phase modulating equations that describe the amplitudes and phases evolution at superharmonic resonance case as

$$
\begin{aligned}
\dot{a}_{1}= & -\frac{1}{2} \mu_{1} a_{1}+\frac{1}{8 \omega} \beta a_{1} a_{2}^{2} \sin \left(\phi_{2}-\phi_{1}\right)+ \\
& \frac{1}{8 \omega} \alpha a_{1} \sin \left(\phi_{1}\right)-\frac{1}{8 \omega} \alpha a_{2} \cos \left(\frac{\phi_{1}+\phi_{2}}{2}\right) ; \\
\dot{a}_{2}= & -\frac{1}{2} \mu_{2} a_{2}-\frac{1}{8 \omega} \beta a_{1}^{2} a_{2} \sin \left(\phi_{2}-\phi_{1}\right)- \\
& \frac{1}{8 \omega} \alpha a_{2} \sin \left(\phi_{2}\right)-\frac{1}{8 \omega} \alpha a_{1} \cos \left(\frac{\phi_{1}+\phi_{2}}{2}\right) ; \\
\dot{\phi}_{1}= & 3 \sigma_{3}-\frac{3}{4 \omega} \beta a_{1}^{2}-\frac{1}{4 \omega} \beta a_{2}^{2} \cos \left(\phi_{2}-\phi_{1}\right)-\frac{1}{2 \omega} \beta a_{2}^{2}+ \\
& \frac{1}{4 \omega} \alpha \cos \left(\phi_{1}\right)+\frac{1}{4 \omega a_{1}} \alpha a_{2} \sin \left(\frac{\phi_{1}+\phi_{2}}{2}\right) ; \\
\dot{\phi}_{2}= & 3 \sigma_{3}-\frac{3}{4 \omega} \beta a_{2}^{2}-\frac{1}{4 \omega} \beta a_{1}^{2} \cos \left(\phi_{2}-\phi_{1}\right)-\frac{1}{2 \omega} \beta a_{1}^{2}- \\
& \frac{1}{4 \omega} \alpha \cos \left(\phi_{2}\right)+\frac{1}{4 \omega a_{2}} \alpha a_{1} \sin \left(\frac{\phi_{1}+\phi_{2}}{2}\right) ;
\end{aligned}
$$

where $\phi_{1}=3 \sigma_{3} t-2 \psi_{1}, \phi_{2}=3 \sigma_{3} t-2 \psi_{2}$. 


\section{STEADY STATE WHIRLING MOTION AND ITS STABILITY}

At steady state vibrations, we have $\dot{a}_{1}=\dot{a}_{2}=\dot{\phi}_{1}=\dot{\phi}_{2}=$ 0 . Substituting this condition into Eqs. (19a)-(19d), (20a)(20d), and (21a)-(21d), we get the steady state whirling amplitude of the system at the three considered resonant cases. For example, the steady state motion at the primary resonance can be obtained from Eqs. (19a)-(19d) as follows:

$$
\begin{gathered}
-\frac{\mu_{1}}{2} a_{1}+\frac{\beta}{8 \omega} a_{1} a_{2}^{2} \sin \left(2 \phi_{2}-2 \phi_{1}\right)+\frac{f \Omega^{2}}{2 \omega} \sin \left(\phi_{1}\right)+ \\
\frac{\alpha}{4 \omega} a_{1} \sin \left(2 \phi_{1}\right)-\frac{\alpha}{4 \omega} a_{2} \cos \left(\phi_{1}+\phi_{2}\right)=0 ; \\
-\frac{\mu_{2}}{2} a_{2}-\frac{\beta}{8 \omega} a_{1}^{2} a_{2} \sin \left(2 \phi_{2}-2 \phi_{1}\right)-\frac{f \Omega^{2}}{2 \omega} \cos \left(\phi_{2}\right)- \\
\frac{\alpha}{4 \omega} a_{2} \sin \left(2 \phi_{2}\right)-\frac{\alpha}{4 \omega} a_{1} \cos \left(\phi_{1}+\phi_{2}\right)=0 ; \\
\sigma_{1}-\frac{3 \beta a_{1}^{2}}{8 \omega}-\frac{\beta a_{2}^{2}}{8 \omega} \cos \left(2 \phi_{2}-2 \phi_{1}\right)-\frac{\beta a_{2}^{2}}{4 \omega}+\frac{f \Omega^{2}}{2 a_{1} \omega} \cos \left(\phi_{1}\right)+ \\
\frac{\alpha}{4 \omega} \cos \left(2 \phi_{1}\right)+\frac{\alpha a_{2}}{4 a_{1} \omega} \sin \left(\phi_{1}+\phi_{2}\right)=0 ; \\
\sigma_{1}-\frac{3 \beta a_{2}^{2}}{8 \omega}-\frac{\beta a_{1}^{2}}{8 \omega} \cos \left(2 \phi_{2}-2 \phi_{1}\right)-\frac{\beta a_{1}^{2}}{4 \omega}+\frac{f \Omega^{2}}{2 a_{2} \omega} \sin \left(\phi_{2}\right)- \\
\frac{\alpha}{4 \omega} \cos \left(2 \phi_{2}\right)+\frac{\alpha a_{1}}{4 a_{2} \omega} \sin \left(\phi_{1}+\phi_{2}\right)=0 .
\end{gathered}
$$

Since there is no closed-form solution for Eqs. (22a)-(22d), Newton-Raphson methods are utilized to obtain the numerical solution. Additionally, the solution stability was determined by examining the eigenvalues of the Jacobian matrix on the right-hand side of Eqs. (19a)-(19d) according to the Lyapunov indirect method. ${ }^{24}$ To obtain the Jacobian matrix, let $a_{10}, a_{20}$, $\phi_{10}$, and $\phi_{20}$ be the steady state solution of Eqs. (19a)-(19d). Therefore, we need to examine the behaviour of small deviations from that solution. Thus, we assume that

$$
\begin{aligned}
& a_{1}=a_{11}+a_{10} ; \quad a_{2}=a_{21}+a_{20} ; \\
& \phi_{1}=\phi_{11}+\phi_{10} ; \quad \phi_{2}=\phi_{21}+\phi_{20} ; \\
& \Downarrow \\
& \dot{a}_{1}=\dot{a}_{11} ; \quad \dot{a}_{2}=\dot{a}_{21} ; \quad \dot{\phi}_{1}=\dot{\phi}_{11} ; \quad \dot{\phi}_{2}=\dot{\phi}_{21} ;
\end{aligned}
$$

where $a_{11}, a_{21}, \phi_{11}, \phi_{21}$ are perturbations that are assumed to be small compared to $a_{10}, a_{20}, \phi_{10}$, and $\phi_{20}$. Substituting Eq. (23) into Eqs. (19a)-(19d) and expanding for small $a_{11}$, $a_{21}, \phi_{11}$, and $\phi_{21}$ with keeping the linear terms only, yields

$$
\left(\begin{array}{c}
\dot{a}_{11} \\
\dot{a}_{21} \\
\dot{\phi}_{11} \\
\dot{\phi}_{21}
\end{array}\right)=\left(\begin{array}{llll}
\delta_{11} & \delta_{12} & \delta_{13} & \delta_{14} \\
\delta_{21} & \delta_{22} & \delta_{23} & \delta_{24} \\
\delta_{31} & \delta_{32} & \delta_{33} & \delta_{34} \\
\delta_{41} & \delta_{42} & \delta_{43} & \delta_{44}
\end{array}\right)\left(\begin{array}{l}
a_{11} \\
a_{21} \\
\phi_{11} \\
\phi_{21}
\end{array}\right) .
$$

The above matrix is the Jacobian matrix and their coefficients $\delta_{k j}\{k, j=1,2,3,4\}$ at the three considered resonant cases are given in the appendix. The characteristic equations of the Jacobian matrix can be given as follows:

$$
\lambda^{4}+\Delta_{1} \lambda^{3}+\Delta_{2} \lambda^{2}+\Delta_{3} \lambda+\Delta_{4}=0
$$

where $\lambda$ denotes the eigenvalues of the matrix, $\Delta_{1}, \Delta_{2}, \Delta_{3}$, and $\Delta_{4}$ are the coefficient of Eq. (25), which is given in the appendix. According to Routh-Hurwitz criterion, the necessary and sufficient conditions for the solution of Eqs. (19a)-(19d) to be asymptotically stable are

$$
\begin{gathered}
\Delta_{1}>0 ; \quad \Delta_{1} \Delta_{2}-\Delta_{3}>0 \\
\Delta_{3}\left(\Delta_{1} \Delta_{2}-\Delta_{3}\right)-\Delta_{1}^{2} \Delta_{4}>0 ; \quad \Delta_{4}>0 .
\end{gathered}
$$

Based on Eqs. (22a)-(22d), the steady state solutions are obtained, and corresponding to the conditions Eq. (26), the stability criteria are checked at primary resonance case. The same procedure for stability analysis has been applied to the other resonance cases (i.e. sub- and super-harmonic).

\section{RESULTS AND DISCUSSIONS}

In this section, unless stated otherwise, the vibrations of a nonlinear Jeffcott rotor system with a cracked shaft is investigated at the following values of the system parameters: $f=$ 0.025, $\alpha=0.05, \beta=0.05, \omega=\sqrt{1-\alpha}, \mu_{1}=\mu_{2}=0.01$, $\gamma=0$. Solid lines correspond to stable solutions, while dashed lines correspond to unstable ones. Some of the obtained bifurcation diagrams have been validated numerically by solving Eqs. (5a)-(5b) using ODE45 MATLAB solver. The numerical results are illustrated as small-circles when the bifurcation parameter (i.e. $\sigma_{1}, \sigma_{2}, \sigma_{3}, \alpha, \gamma$, or $f$ ) is varied from small to large values and as big-dots when it is varied from the large to small values.

\subsection{Primary Resonance $\left(\Omega=\omega+\sigma_{1}\right)$}

Figure 3 illustrates the spinning speed-response curve when the disc eccentricity $f=0.025$ and $\alpha=0$ (i.e. without crack). It is noticed from the figure, the bending of the curves to right due to the cubic nonlinearity resulting in jump phenomenon and bi-stable whirling orbits in a wide range above the major critical speed $\omega$ (i.e. $\sigma_{1}>0$ ). The spinning speed- response curve of the cracked rotor system at different values of $\alpha$ (i.e. different crack depths) at zero imbalance is shown in Fig. 4. It is clear from the figure that as $\alpha$ increases, both the vibration amplitude and bandwidth around $\sigma_{1}=0$ increase. The influence of both crack and disc eccentricity are displayed in Fig. 5. It is noticed from the figure that the appearance of new hidden stable whirling motions that are not excited during the traditional acceleration or deceleration of the rotating disc, but requires especial initial positions for the disc to be raised. Therefore, it is expected that the cracked rotor system having a nonzero eccentricity can perform three different whirling orbits if its spinning speed $\Omega$ is slightly higher than the major critical speed $\omega$.

In Fig. 6, the influence of the eccentricity on the spinning speed-response curve is illustrated. It is evident from the figure that increasing the eccentricity magnitude, increases both the whirling amplitude and differences between the tri-stable whirling motions. Eccentricity-response curve at different values of $\sigma_{1}$ is depicted in Fig. 7. The figure illustrates that tristable whirling motions begin to emerge when $\sigma_{1} \geq 0.03$. It is worth stating that the generation of a tri-stable whirling motion can be used as indication to the existence of a transverse crack. Figures 8,9 , and 10 are numerically simulating points $p_{1}, p_{2}$, and $p_{3}$, that showed on Figs. $7 \mathrm{~g}$, and $7 \mathrm{~h}$. The figures confirm the presence of three forward whirling orbits at the same spinning speed $(\omega=\omega+0.05)$, which depends on the disc initial position. 

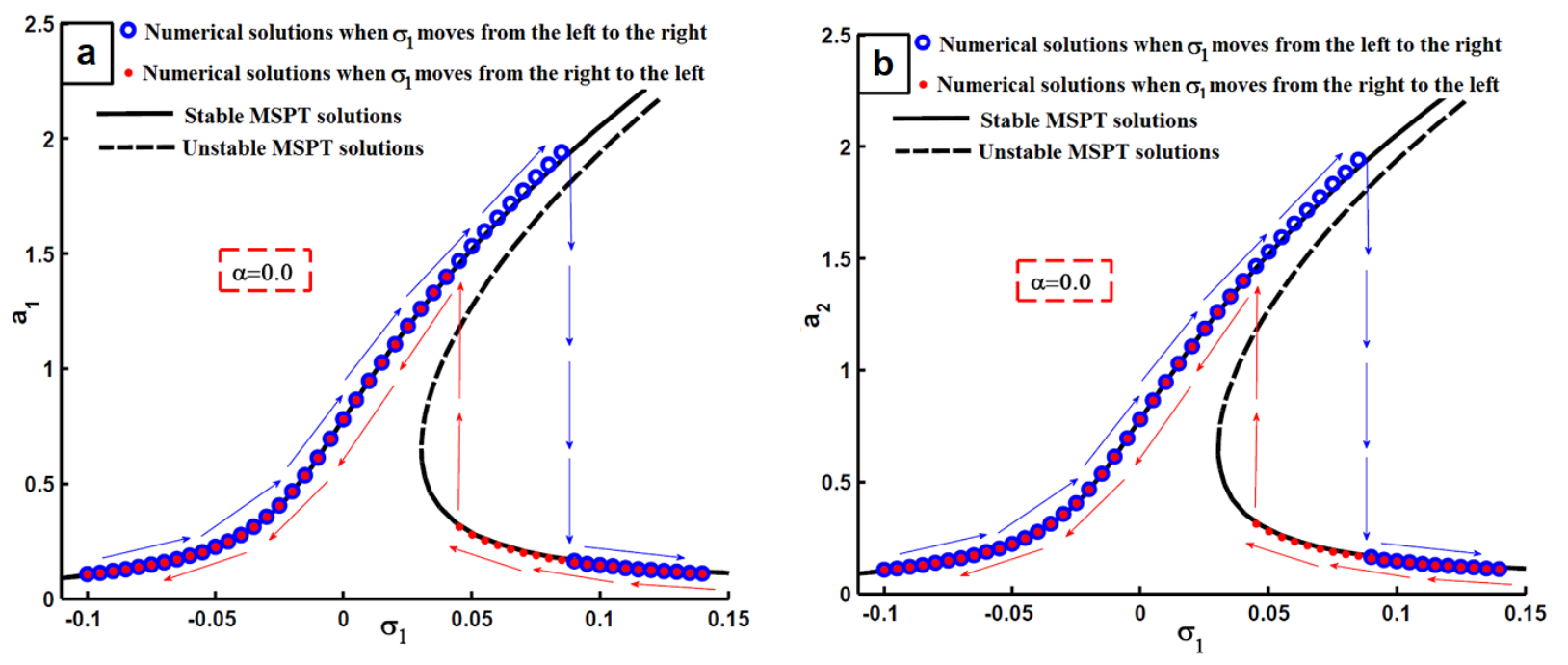

Figure 3. Jeffcott rotor spinning speed-response curve when $\alpha=0.0$ : (a) $y_{1}$-mode, and (b) $y_{2}$-mode.

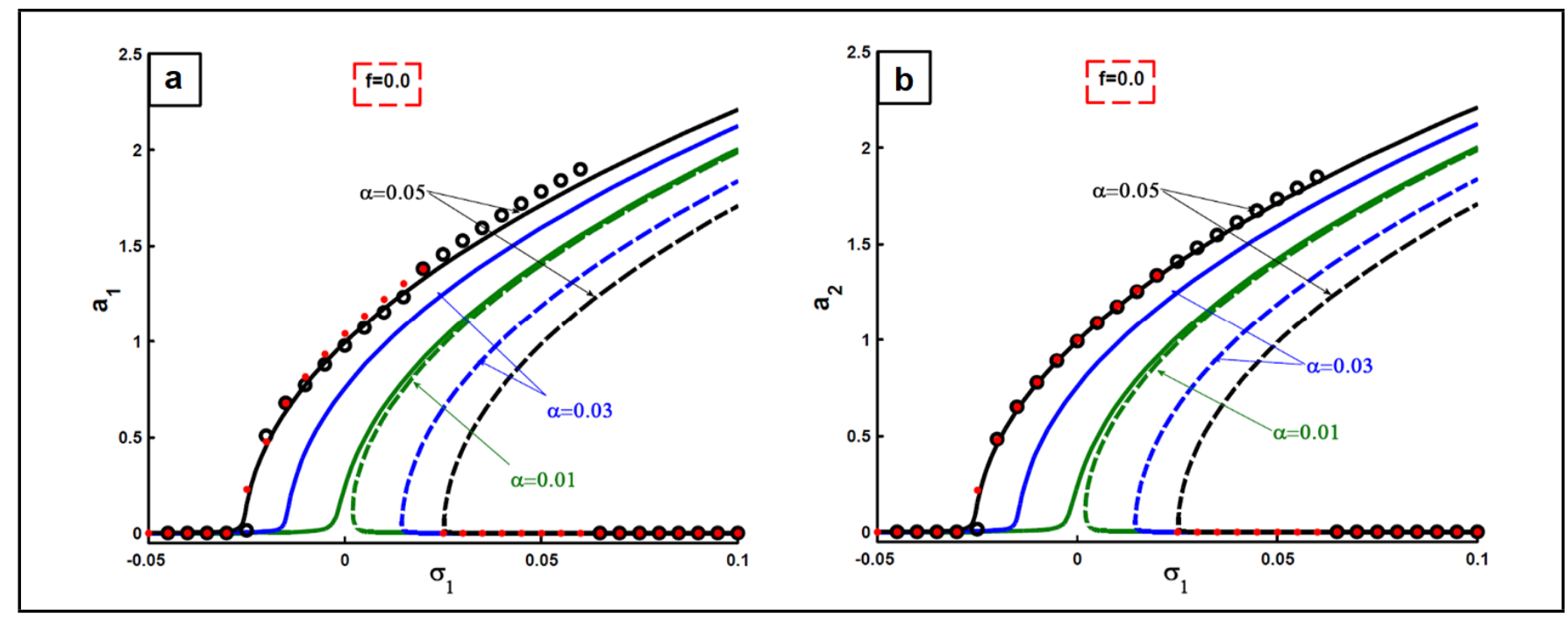

Figure 4. Jeffcott rotor spinning speed-response curve when $f=0.0$ : (a) $y_{1}$-mode, and (b) $y_{2}$-mode.
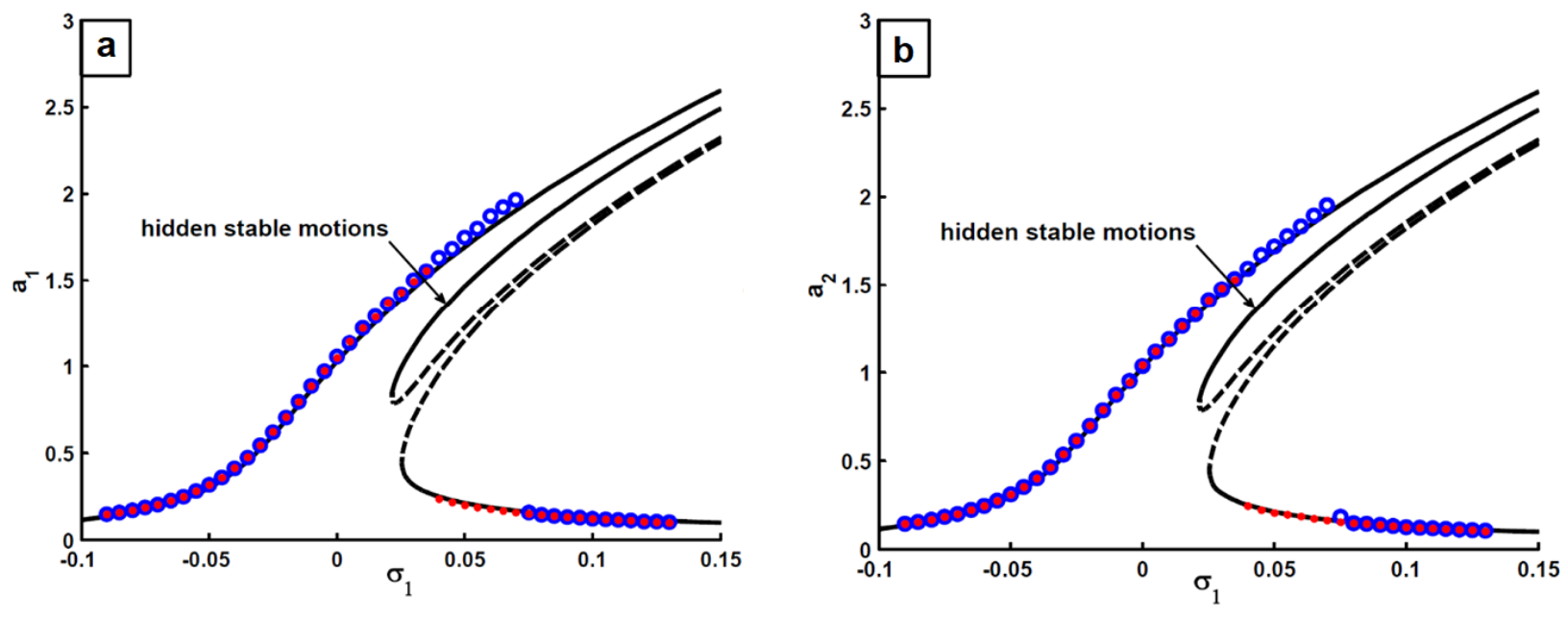

Figure 5. Jeffcott rotor spinning speed-response curve when $f=0.025, \alpha=0.03$ : (a) $y_{1}$-mode, and (b) $y_{2}$-mode. 


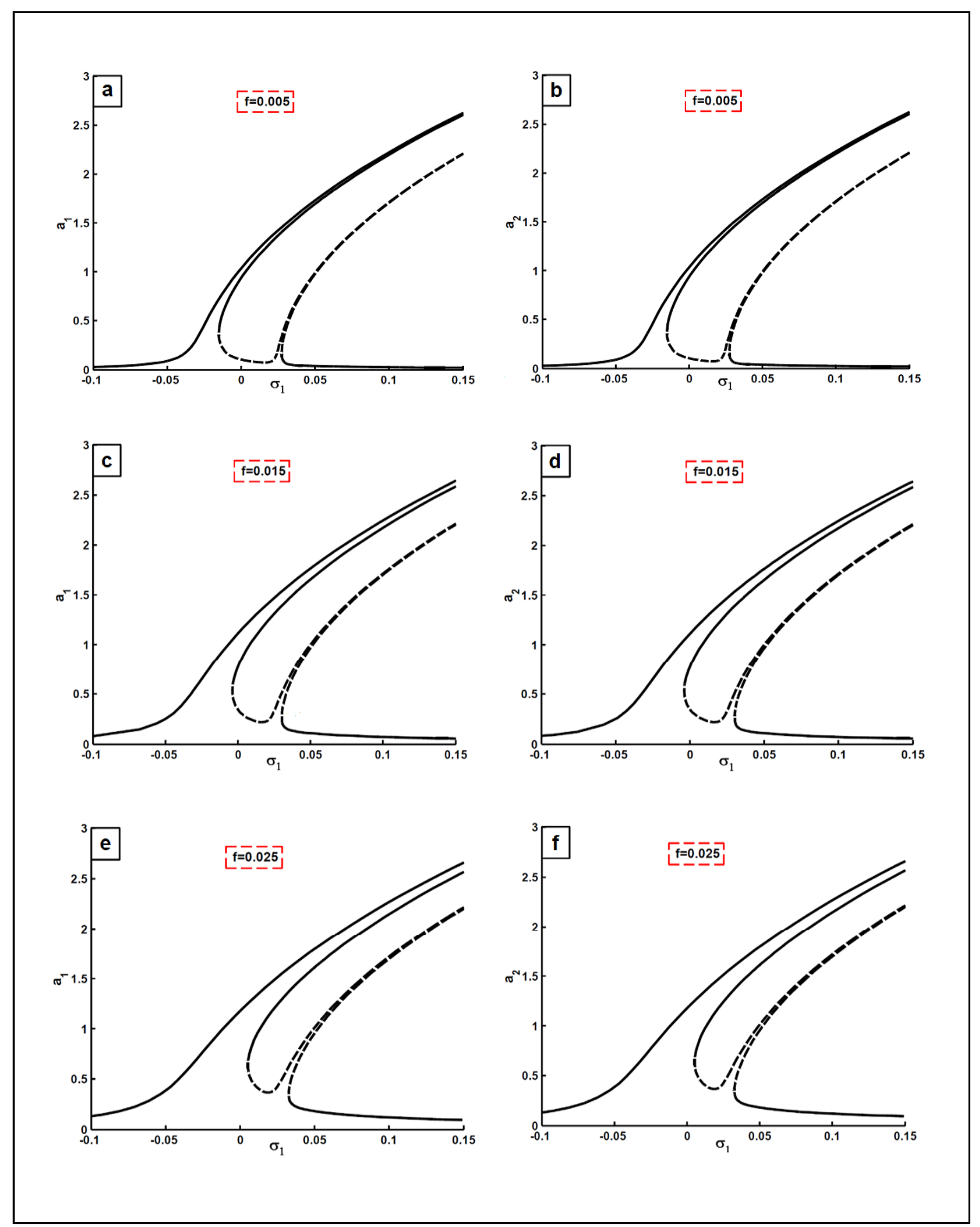

Figure 6. Effect of increasing the eccentricity $f$ on the Jeffcott rotor spinning speed-response curve: (a, c, e) $y_{1}$-mode, and (b, d, f) $y_{2}$-mode. 


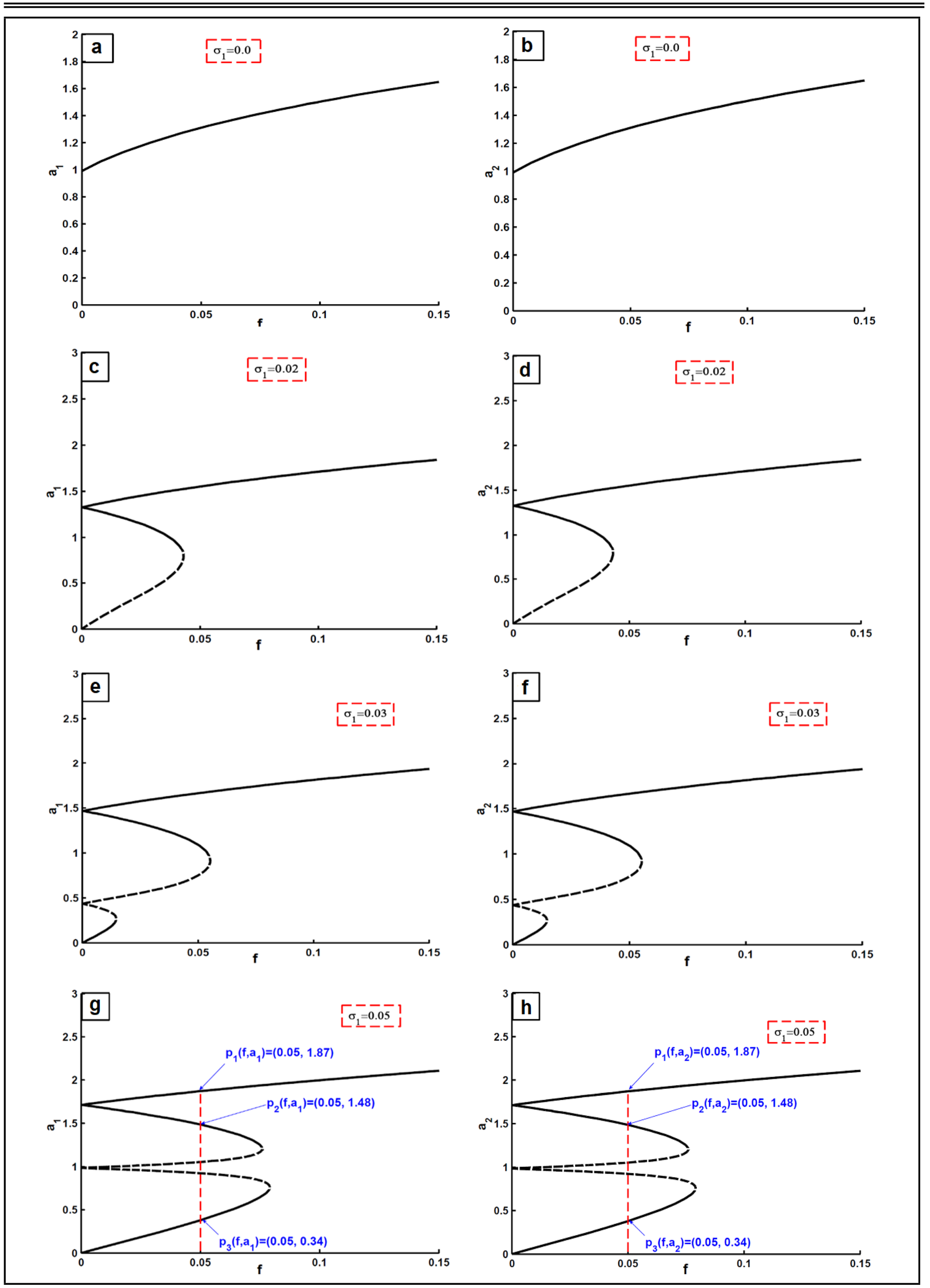

Figure 7. Jeffcott rotor eccentricity-response curve at different spinning speed: (a, c, e, g) $y_{1}$-mode, and (b, d, f, h) $y_{2}$-mode. 


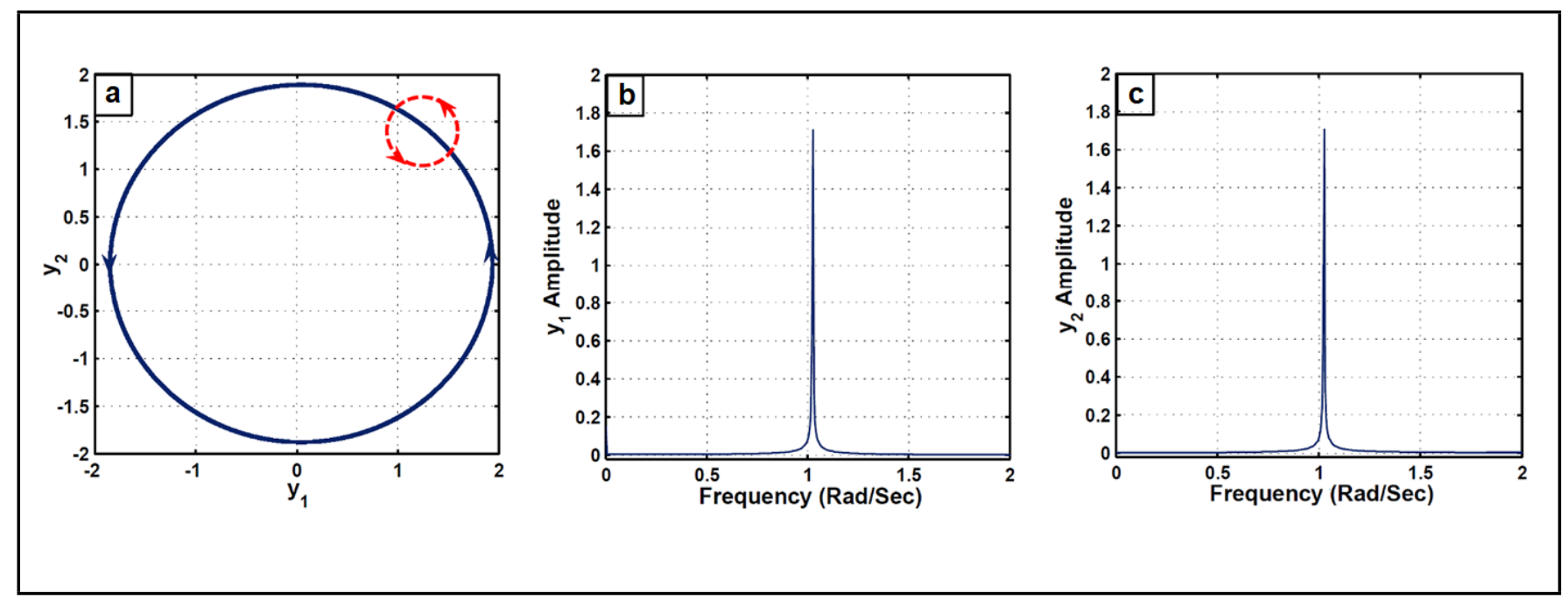

Figure 8. Simulation of point $p_{1}$ on Fig. 7. (i.e. $f=\alpha=0.05, \Omega=\omega+0.05$ ): (a) Jeffcott rotor whirling orbit and (c, d) frequency-spectrum when the disc center $G$ starts from the position $y_{1}(0)=y_{2}(0)=0.75$.

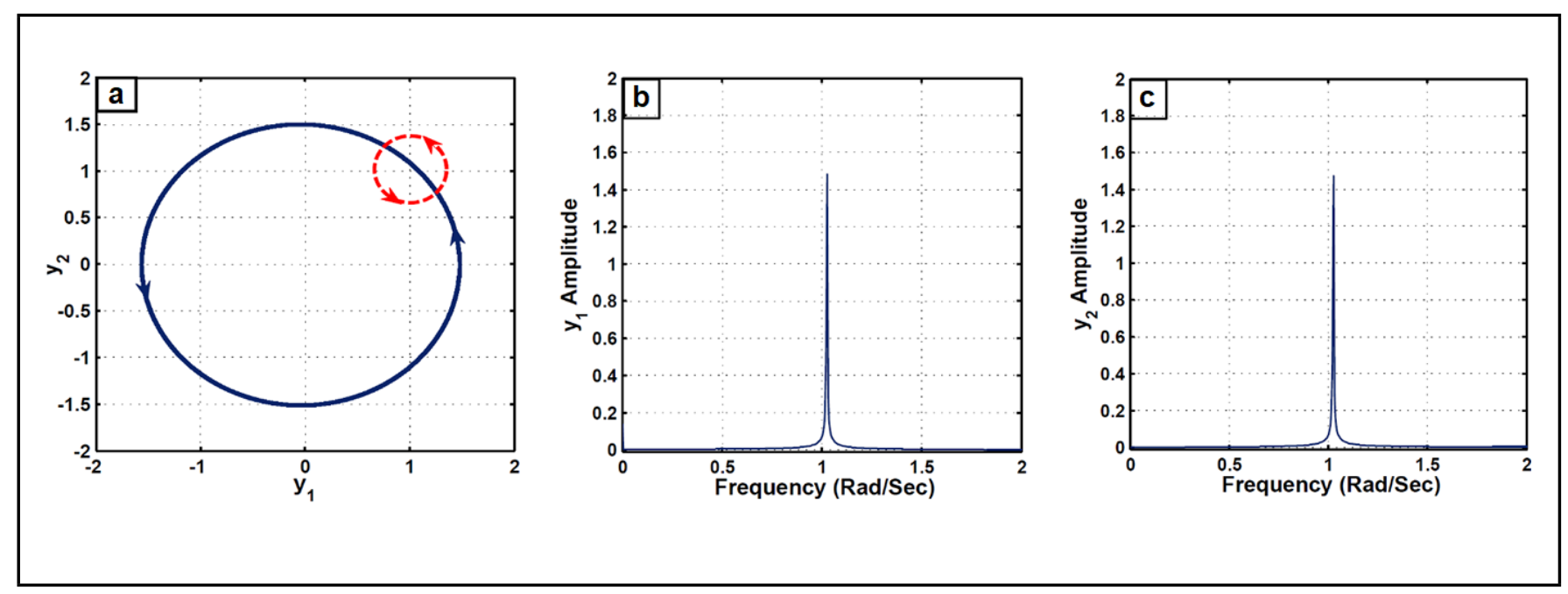

Figure 9. Simulation of point $p_{2}$ on Fig. 7. (i.e. $f=\alpha=0.05, \Omega=\omega+0.05$ ): (a) Jeffcott rotor whirling orbit and (c, d) frequency-spectrum, when the disc center $G$ starts from the position $y_{1}(0)=-1.5, y_{2}(0)=1$.

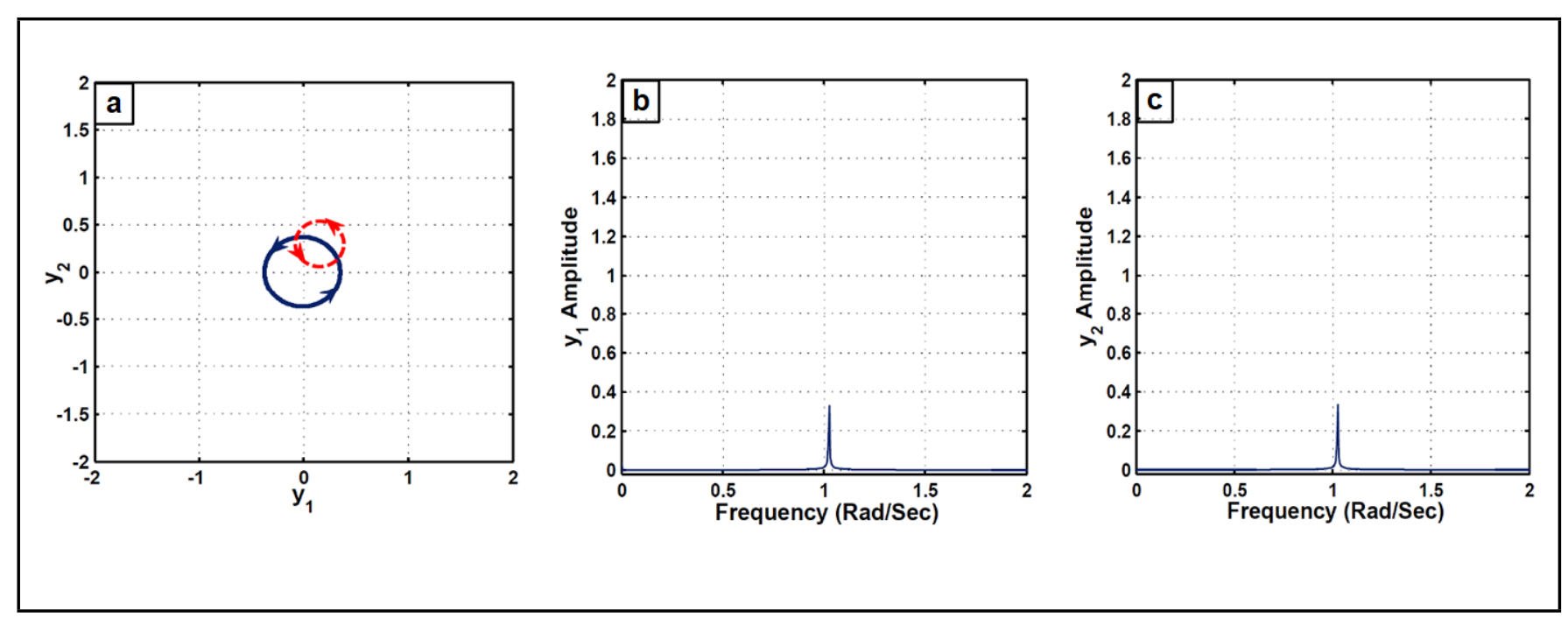

Figure 10. Simulation of point $p_{3}$ on Fig. 7. (i.e. $f=\alpha=0.05, \Omega=\omega+0.05$ ): (a) Jeffcott rotor whirling orbit and (c, d) frequency-spectrum, when the disc center $G$ starts from the position $y_{1}(0)=y_{2}(0)=0.0$. 

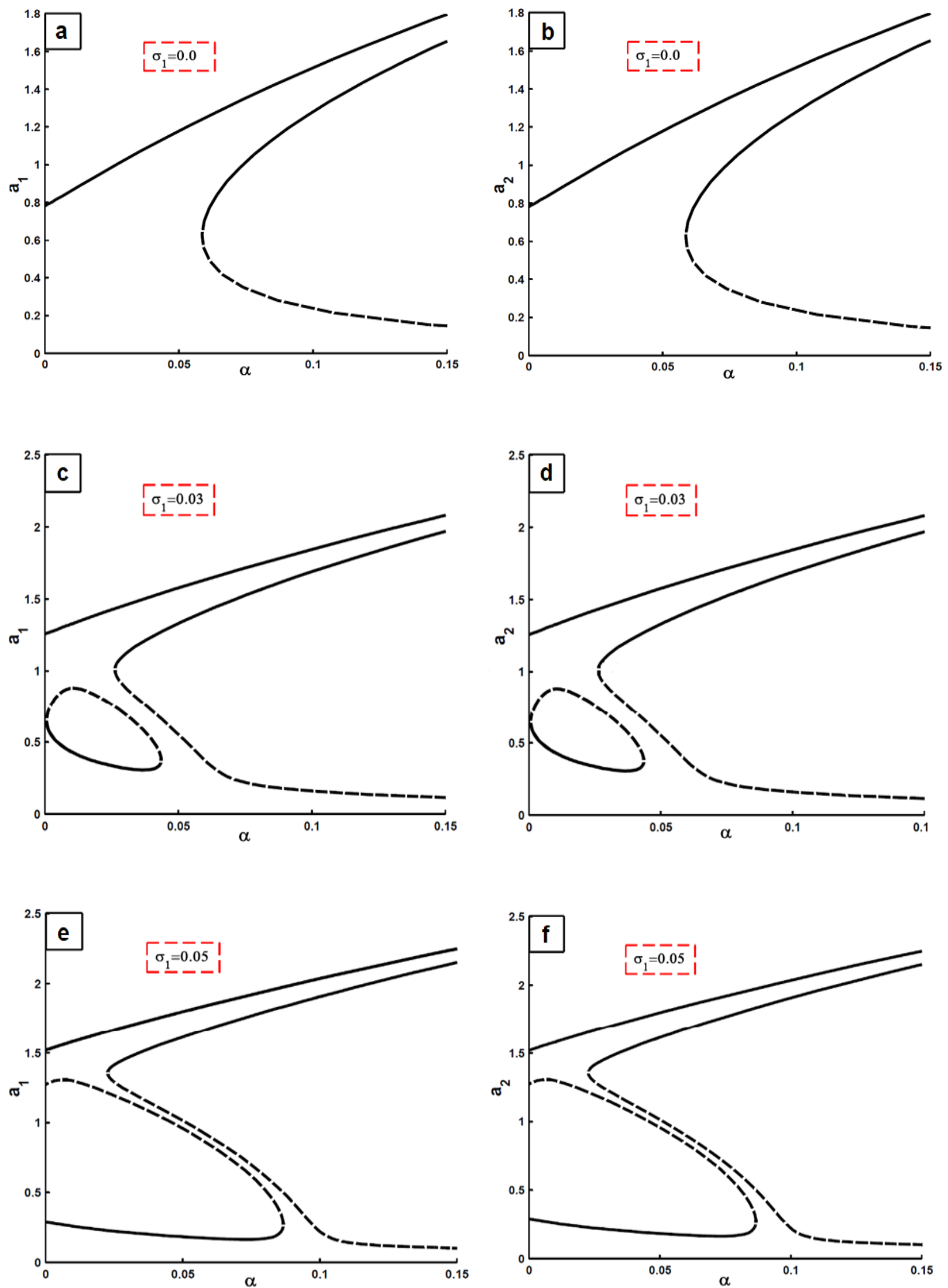

Figure 11. Jeffcott rotor $\alpha$-response curve at different spinning speed $\left(\Omega=\omega+\sigma_{1}\right)$ : (a, c, e) $y_{1}$-mode, and (b, d, f) $y_{2}$-mode. 

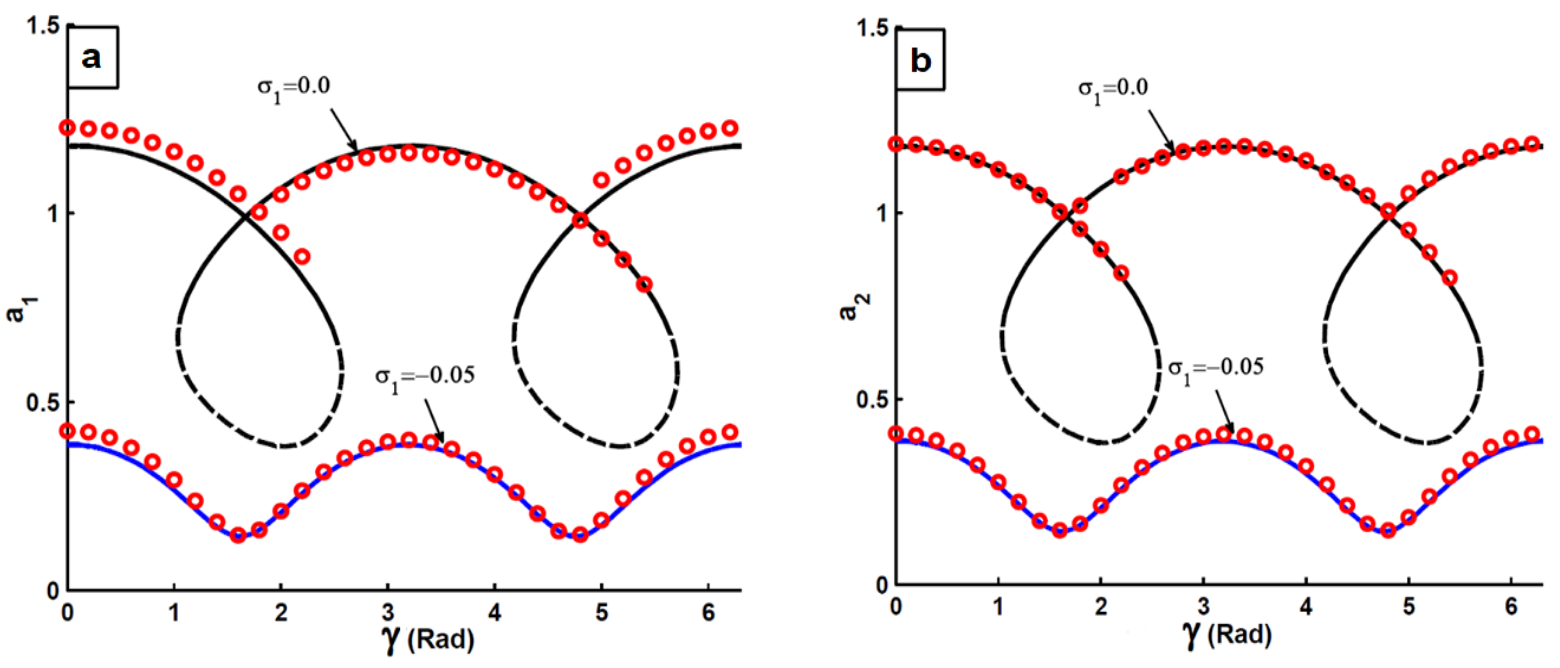

Figure 12. Orientation angle $\gamma$-response curve at $\sigma_{1}=-0.05,0$ : (a) $y_{1}$-mode, and (b) $y_{2}$-mode.
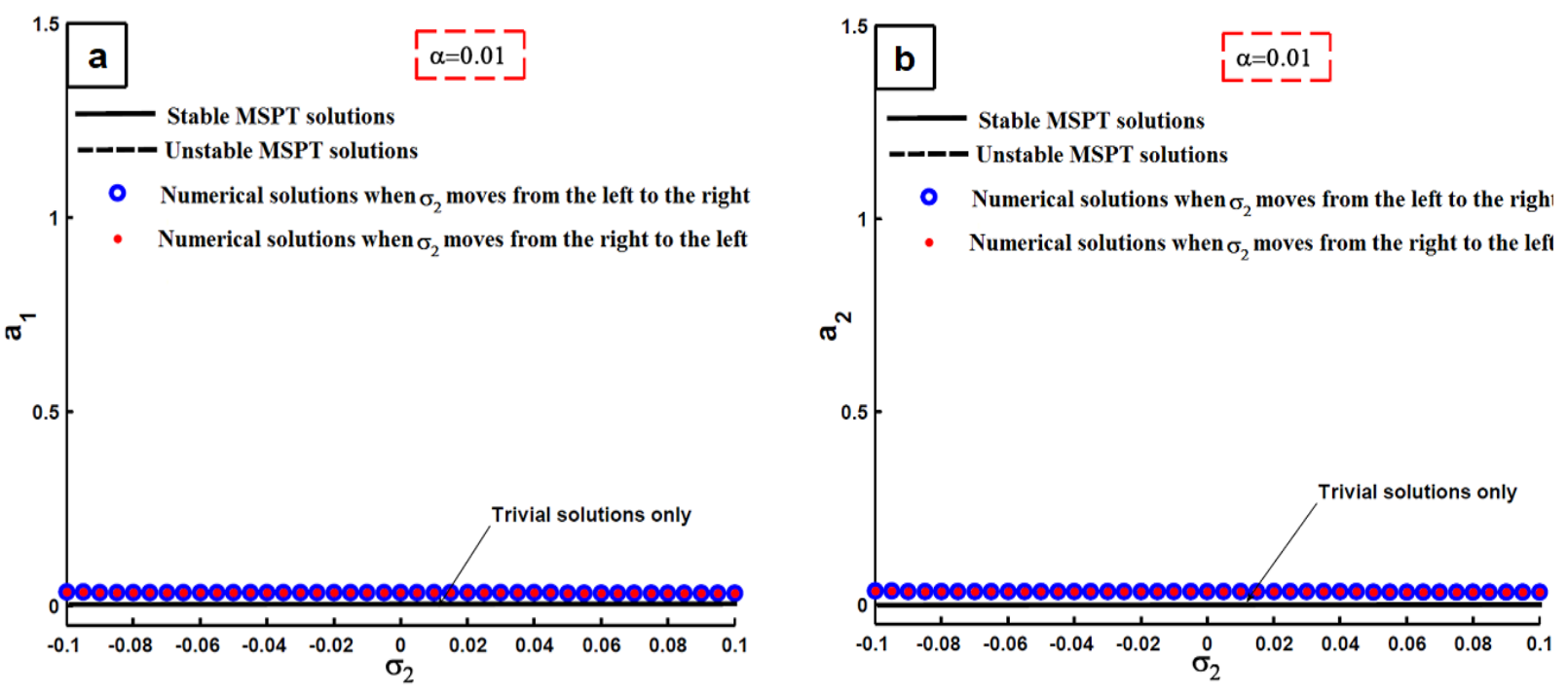

Figure 13. Jeffcott rotor spinning speed-response curve at the resonant case $\left(\Omega \cong 2 \omega+\sigma_{2}\right)$ when $\alpha=0.01$ : (a) $y_{1}$-mode, and (b) $y_{2}$-mode.
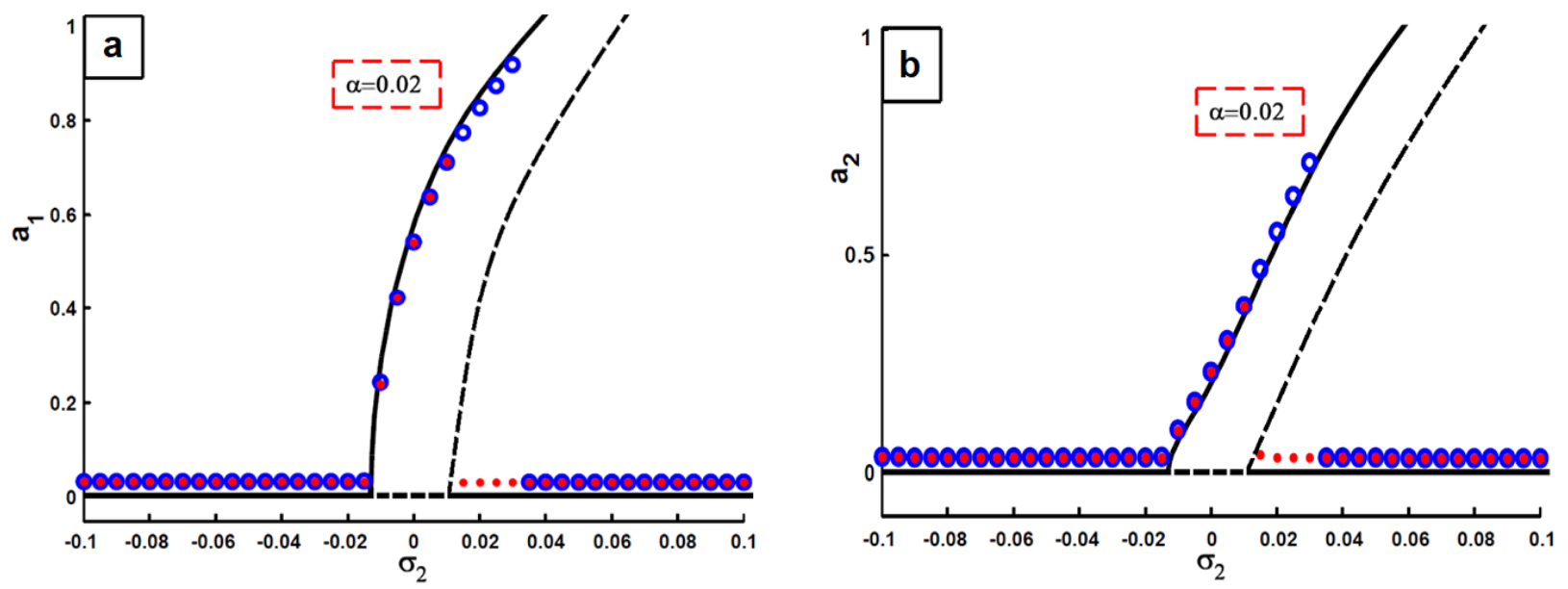

Figure 14. Jeffcott rotor spinning speed-response curve at the resonant case $\left(\Omega \cong 2 \omega+\sigma_{2}\right)$ when $\alpha=0.02$ : (a) $y_{1}$-mode, and (b) $y_{2}$-mode. 
The relative reduction of the linear shaft-stiffness $(\alpha)$ due to the crack is plotted against the response amplitudes in Fig. 11 at different values of the spinning speed. It is clear from the figure that the whirling amplitudes are monotonic increasing functions in $\alpha$, and the existence of the multi-valued solutions depends on both the spinning speed and the relative reduction of the linear shaft-stiffness. In Fig. 12, the orientation angle $(\gamma)$ is plotted versus the response amplitudes. The figure shows that the maximum response amplitude may appear if the crack and imbalance are in the same or opposite directions (i.e. when $\gamma=0^{\circ}$ or $180^{\circ}$ ), and the minimum vibrations occurs if their directions are perpendicular to each other (i.e. when $\gamma=\frac{\pi}{2}$ or $\left.-\frac{\pi}{2}\right)$

\subsection{Sub-Harmonic Resonance $\left(\Omega=2 \omega+\sigma_{2}\right)$}

It is evident from Eqs. (20a)-(20d) that the whirling motions at sub harmonic resonance does not depend on the disc eccentricity and hence the orientation angle. Therefore, the only reason of the whirling motions at $\Omega \cong 2 \omega$ is the exerted parametric force due to time-varying stiffness. Figures 13, 14, and 15 illustrate the spinning speed-response curve at three different values of $\alpha$. It is clear from the figures that the whirling motions have been excited when $\alpha \geq 0.02$ (i.e. $\frac{\Delta k}{k_{1}} \geq 0.08$ ). Moreover, increasing $\alpha$, increases the whirling amplitudes and bends the response curves to the right that leading to bi-stable solutions, and jump phenomenon occurrence.

In Fig. 16, $\alpha$-response curve is showed at different values of the detuning parameter $\sigma_{2}$, while Fig. 17 shows numerical confirmation for one of the obtained curves in Fig. 16. As it is clear from Figs. 13, 14, 15, and 17 that the obtained numerical solutions of Eqs. (5a)-(5b) and acquired analytically results using multiples scale method show an excellent agreement. The whirling orbits and frequency spectrum of the cracked Jeffcott rotor system according to points $p_{1}$ and $p_{2}$ that illustrated in Fig. 17 are given in Figs. 18 and 19, respectively. The two figures show the existence of two stable whirling motions at the same spinning speed (i.e. $\Omega=2 \omega+0.05$ ), one of them is the trivial solution and the other has a considerable whirling amplitude.

\subsection{Super-Harmonic Resonance $\left(\Omega=\frac{2}{3} \omega+\sigma_{3}\right)$}

The evolution of whirling amplitudes and their phase angles does not depend on the disc eccentricity and the orientation angle at the resonance case $\Omega \cong \frac{2}{3} \omega$ as it clear from Eqs.(21a)-(21d), but only on the parametric force $\alpha$ resulting from the shaft crack. The spinning speed-response curve at three different values of $\alpha$ is illustrated in Figs. 20, 21, and 22. It is noticed from the figures that the whirling motion does not arise unless $\alpha \geq 0.03$ (i.e. $\frac{\Delta k}{k_{1}} \geq 0.12$ ), and has a narrow bandwidth around $\sigma_{3}=0$ compared to the whirling motion at the sub-harmonic resonant case (i.e. Figs. 13, 14 and 15). The system whirling orbit and frequency spectrum are numerically simulated in Figs. 23 and 24 according to points $P_{1}$ and $P_{2}$ that displayed on Fig. 22. Frequency spectrum shown in Fig. 23 confirms that the forced oscillation with the harmonic and super-harmonic components have been raised, which resulting in more than one loop whirling orbit.

\section{CONCLUSIONS}

This research provides clear understanding and insight analysis for the nonlinear dynamics of a vertically supported Jeffcott rotor system having a transversely cracked shaft. An approximate analytical solution was conducted to the system equations of motion, and numerical confirmations for the obtained results were carried out. The stiffness nonlinearity, the disc imbalance, the breathing crack mechanism, and the orientation angle are included in the system modelling. The analyses illustrated the raising of three resonance cases that are primary, sub-, and super-harmonic. Bifurcation analyses were performed which provided global expectations to the system response at wide range of its parameters. The evolution of the reported resonance cases have been investigated. Accordingly, this work may provide a possible basis for a cracked shafts diagnosis. Based on the above discussion, we may conclude the following:

1. At primary resonance and when the spinning speed is slightly higher than the major critical speed $\omega$, it is noticed the existence of two stable whirling orbits for noncracked rotor system, and three stable whirling orbits for the cracked rotor-system, which one of them was named "hidden-motion".

2. Despite of the qualitative changes in the system dynamics due to the shaft crack at primary resonance case, the accurate detection of a crack may not be successfully achieved due to the effect of the disc imbalance.

3. The whirling oscillations at sub- and super-harmonic resonance cases are independent of both the disc eccentricity and the orientation angle $\gamma$, while the main reason of their excitations is the exerted parametric force due to the shaft-crack.

4. The evolution of the whirling motion at sub- and superharmonic resonance is a clear indication of the transverse crack presence on the rotating shaft.

5. High sensitivity of the sub-harmonic resonance to the crack presence, and the relative wide bandwidth around $\sigma_{2} \cong 0$, make this resonance case the optimal to be utilized for the crack diagnosis in the rotating machines.

6. In contrast to the horizontally supported Jeffcott rotor system (Cheng, et al. ${ }^{5}$ ), at synchronous whirling (i.e. $\Omega \cong \omega$ ) the minimum vibration amplitude occurs when the orientation angle $\gamma \cong \pm \frac{\pi}{2}$, and the maximum vibration amplitudes appear at $\gamma \cong 0^{\circ}$ or $\pi$.

7. All noticed vibrational motions are forward whirling orbits and no backward whirling motions have been reported.

Compared to the above-mentioned articles, Jun, et al. ${ }^{4}$ concluded that the residual imbalance has a little influence on the sub-harmonic resonance, which made it the best case for crack detection purposes. Cheng, et al. ${ }^{5}$ studied numerically the effects of the breathing crack and the imbalance orientation angle on the dynamical behaviour of a horizontally supported Jeffcott rotor system. They reported that the diagnosis of a 

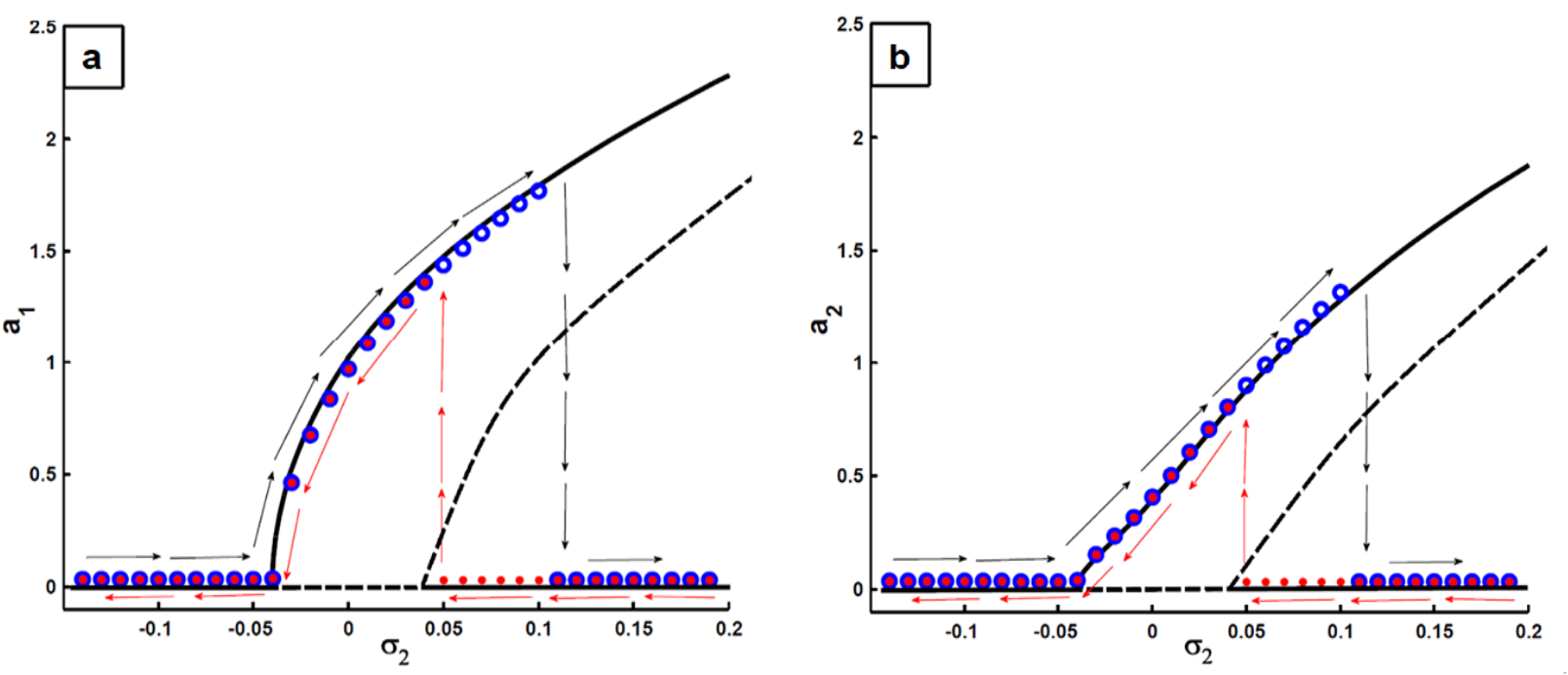

Figure 15. Jeffcott rotor spinning speed-response curve at the resonant case $\left(\Omega \cong 2 \omega+\sigma_{2}\right)$ when $\alpha=0.05$ : (a) $y_{1}$-mode, and (b) $y_{2}$-mode.

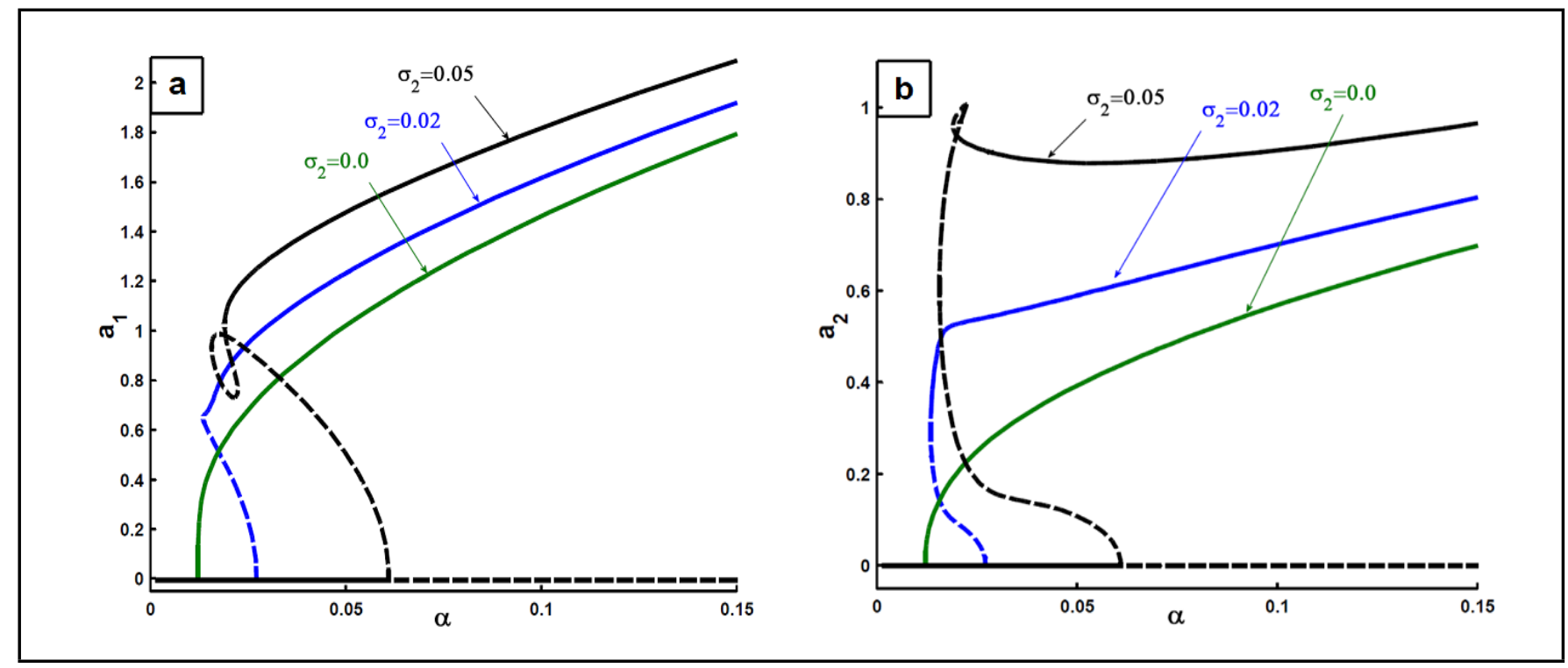

Figure 16. Jeffcott rotor $\alpha$-response curve at different spinning speed $\left(\Omega=2 \omega+\sigma_{2}\right)$ : (a) $y_{1}$-mode, and (b) $y_{2}$-mode.
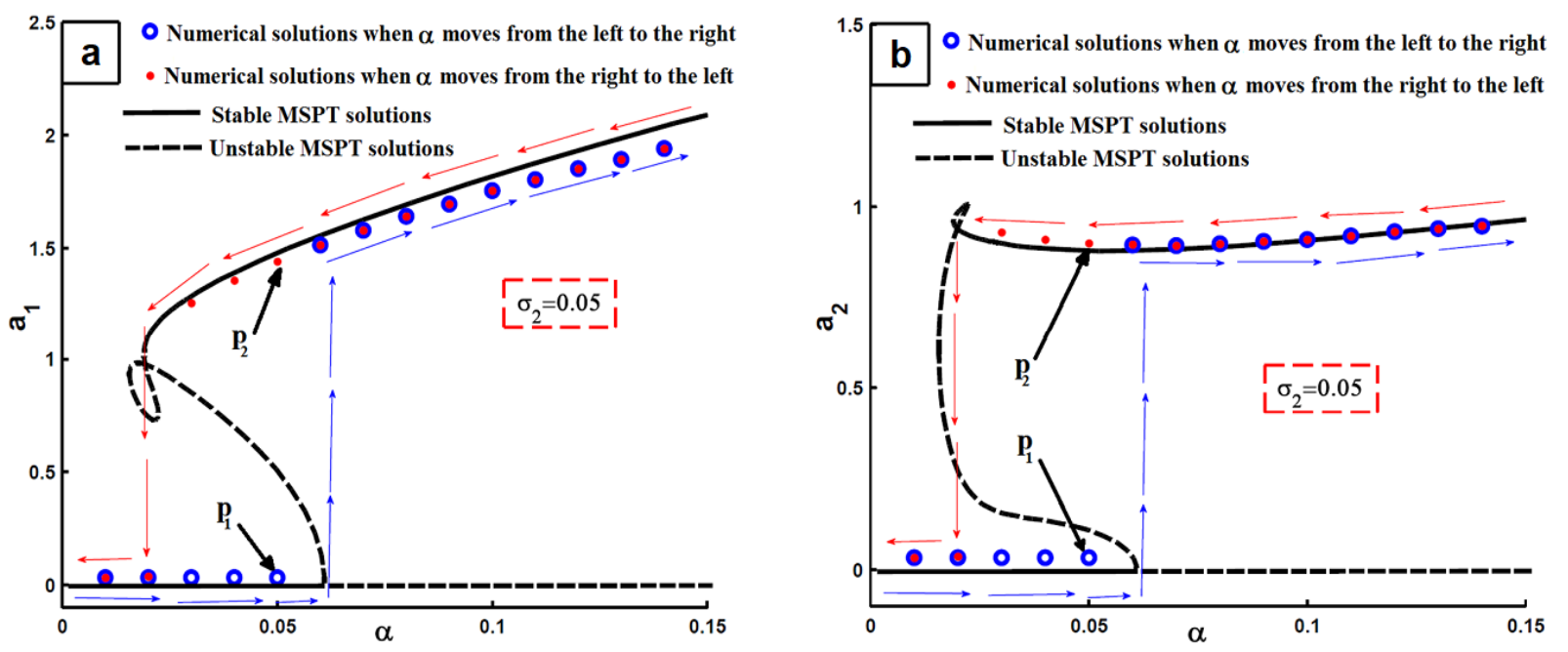

Figure 17. Numerical validations of Fig. 16 at $\sigma_{2}=0.05$ : (a) $y_{1}$-mode, and (b) $y_{2}$-mode. 


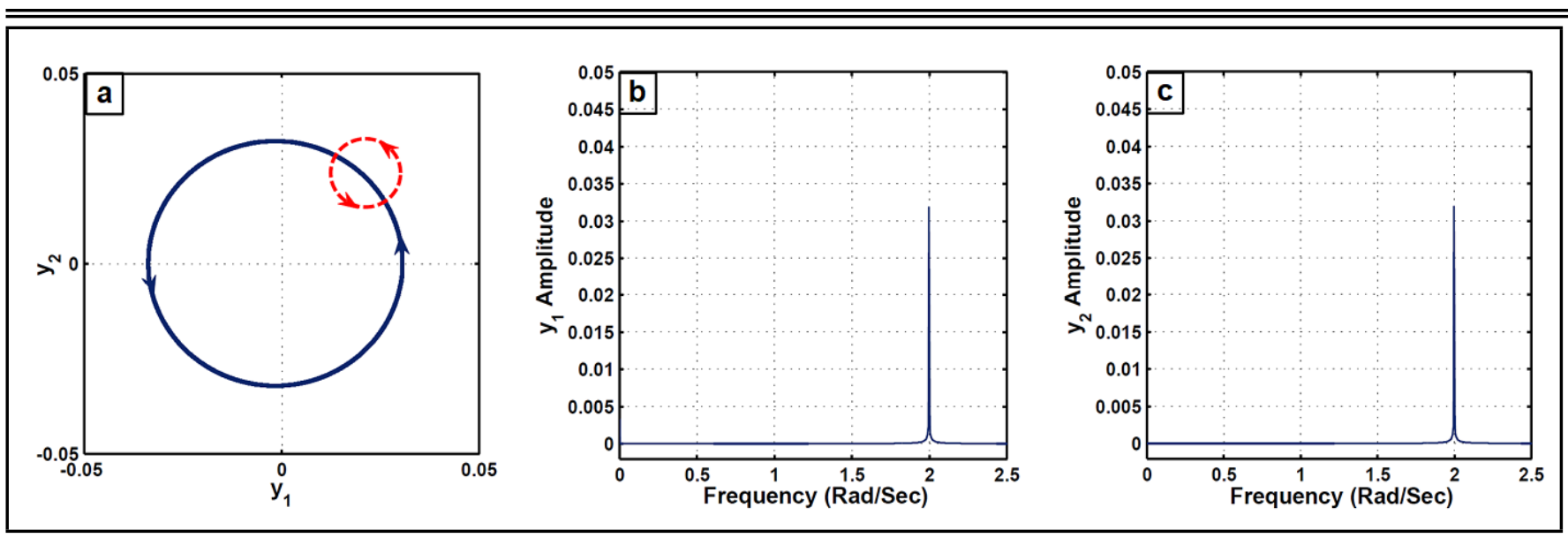

Figure 18. Simulation of point $p_{1}$ on Fig. 17. (i.e. $\alpha=0.05, \Omega=2 \omega+0.05$ ): (a) Jeffcott rotor whirling orbit and (c, d) frequency-spectrum when the disc center $G$ starts rotation from the position $y_{1}(0)=y_{2}(0)=0.0$.

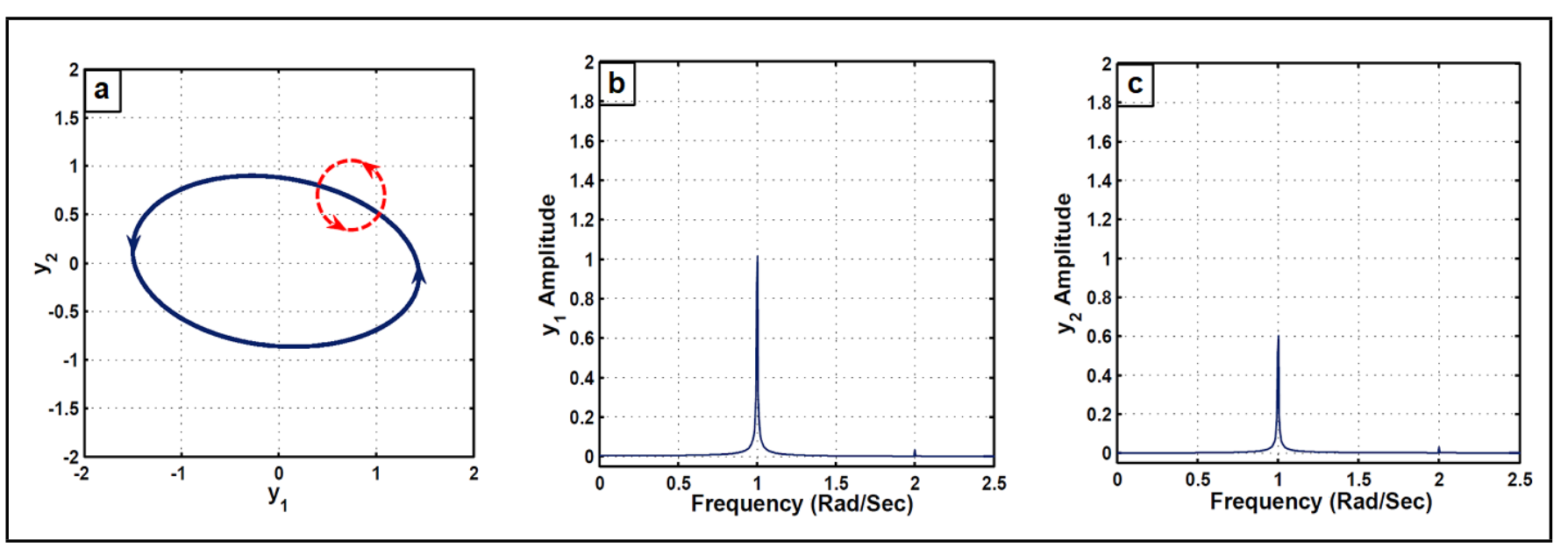

Figure 19. Simulation of point $p_{2}$ on Fig. 17. (i.e. $\alpha=0.05, \Omega=2 \omega+0.05$ ): (a) Jeffcott rotor whirling orbit and (c, d) frequency-spectrum when the disc center $G$ starts rotation from the position $y_{1}(0)=y_{2}(0)=0.5$.

cracked rotor according to the system dynamic at primary resonance case might lead to incorrect data, which is consistent with the results obtained in our work. Sinou ${ }^{7}$ investigated influence of the shaft crack on the Jeffcott rotor stability at $\frac{1}{2}$, $\frac{1}{3}$ super-harmonic resonance case. The author showed that the system performs two or three whirling orbits per revolution when the spinning speed is one-half or one-third of the critical speed. In Sinou and Lees, ${ }^{8}$ Sinou, ${ }^{9}$ and Hou, et al. ${ }^{18}$ the authors concluded that the increasing of the whirling amplitude at the primary resonance or the evolution of a whirling motion at $\frac{1}{2}, \frac{1}{3}$ super-harmonic resonance is an indication of the shaft crack. Within this paper, the analysis illustrated that the $\frac{2}{3}$ super-harmonic and $\frac{2}{1}$ sub-harmonic resonance cases are excited due to the cracked shaft only, and their excitation magnitudes are independent of the disc eccentricity and hence the orientation angle. In addition, the sub-harmonic resonance case is more sensitive than the super-harmonic resonance to the crack presence, which makes it the optimal for crack detection purposes in a vertically supported Jeffcott rotor system.

\section{REFERENCES}

1 Wauer J. On the dynamics of cracked rotors: literature survey, Applied Mechanics Reviews, 43, 13-17, (1990). https://dx.doi.org/10.1115/1.3119157
2 Dimarogonas A. Vibration of cracked structures: a state of the art review, Engineering Fracture Mechanics, 55, 831-857, (1996). https://dx.doi.org/10.1016/00137944(94)00175-8

3 Gasch R. A survey of the dynamic behaviour of a simple rotating shaft with a transverse crack, Journal of Sound and Vibration, 160 (2), 313-332, (1993). https://dx.doi.org/10.1006/jsvi.1993.1026

4 Jun, O. S., Eun, H. J., Earmme, Y. Y., and Lee, C. W. Modelling and vibration analysis of a simple rotor with breathing crack, Journal of Sound and Vibration, 155, 273-290, (1992). https://dx.doi.org/10.1016/0022-460X(92)90511-U

5 Cheng, L., Li, N., Chen, X. F., and He, Z. J. The influence of crack breathing and imbalance orientation angle on the characteristics of the critical speed of a cracked rotor, Journal of Sound and Vibration, 330, 2031-2048, (2011). https://dx.doi.org/10.1016/j.jsv.2010.11.012

6 Sinou, J. J. and Lees, A. W. The influence of cracks in rotating shafts, Journal of Sound and Vibration, 285, 10151037, (2005). https://dx.doi.org/10.1016/j.jsv.2004.09.008

7 Sinou, J. J. Effects of a crack on the stability of a non-linear rotor system, International Journal 


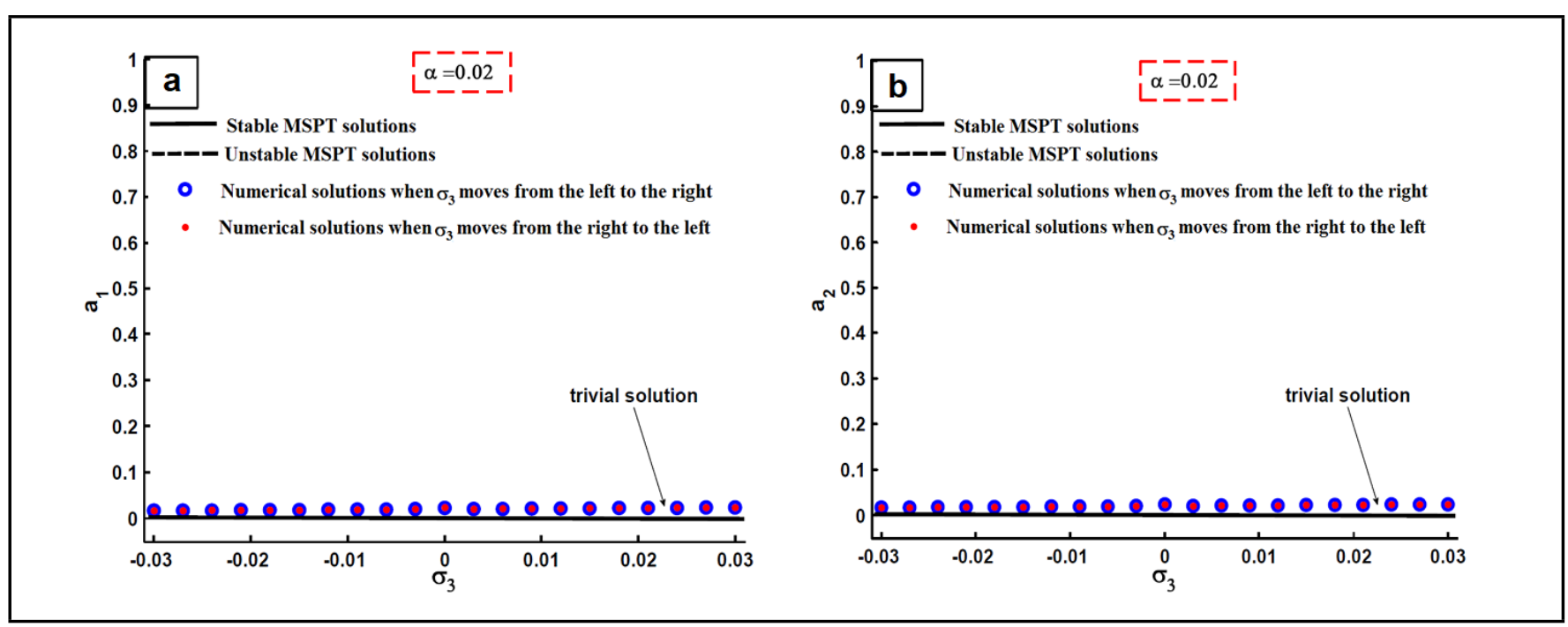

Figure 20. Jeffcott rotor spinning speed-response curve at the resonant case $\left(\Omega \cong \frac{2}{3} \omega+\sigma_{3}\right)$ when $\alpha=0.02$ : (a) $y_{1}$-mode, and (b) $y_{2}$-mode.
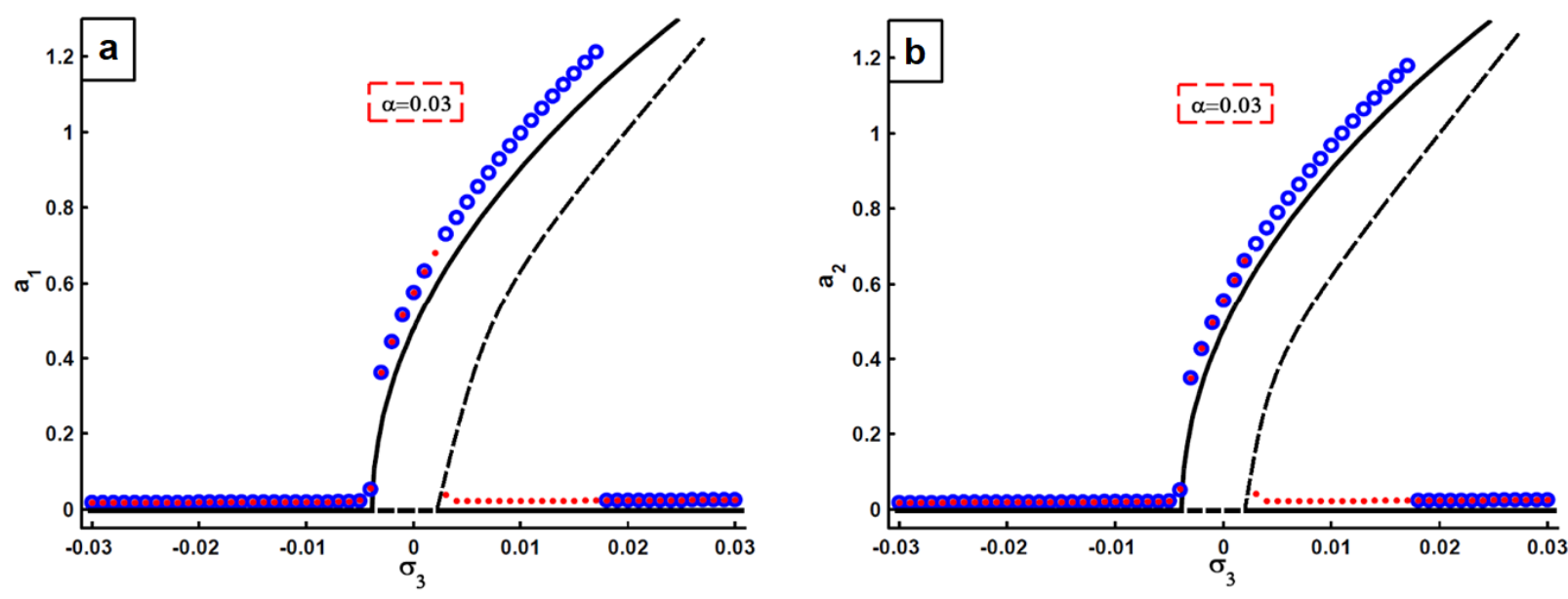

Figure 21. Jeffcott rotor spinning speed-response curve at the resonant case $\left(\Omega \cong \frac{2}{3} \omega+\sigma_{3}\right)$ when $\alpha=0.03$ : (a) $y_{1}$-mode, and (b) $y_{2}$-mode.
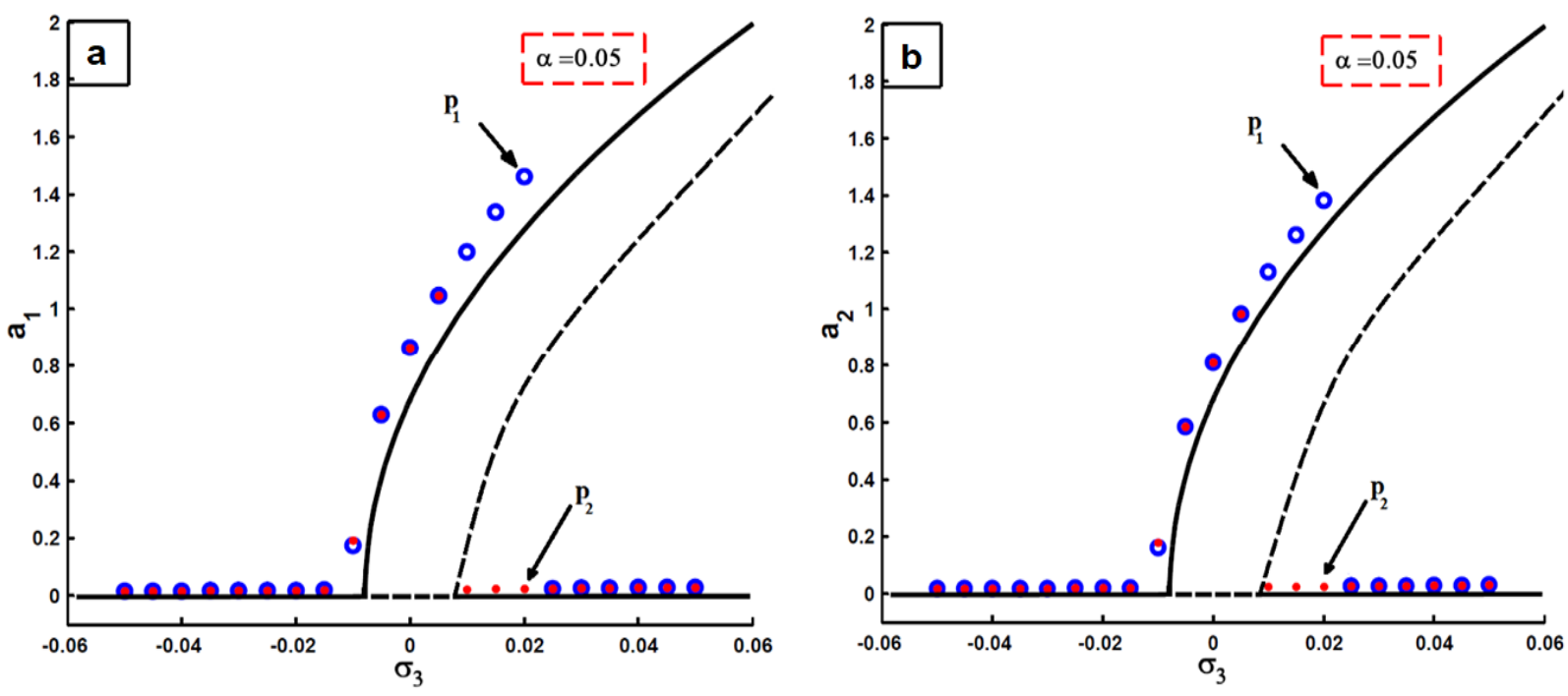

Figure 22. Jeffcott rotor spinning speed-response curve at the resonant case $\left(\Omega \cong \frac{2}{3} \omega+\sigma_{3}\right)$ when $\alpha=0.05$ : (a) $y_{1}$-mode, and (b) $y_{2}$-mode. 


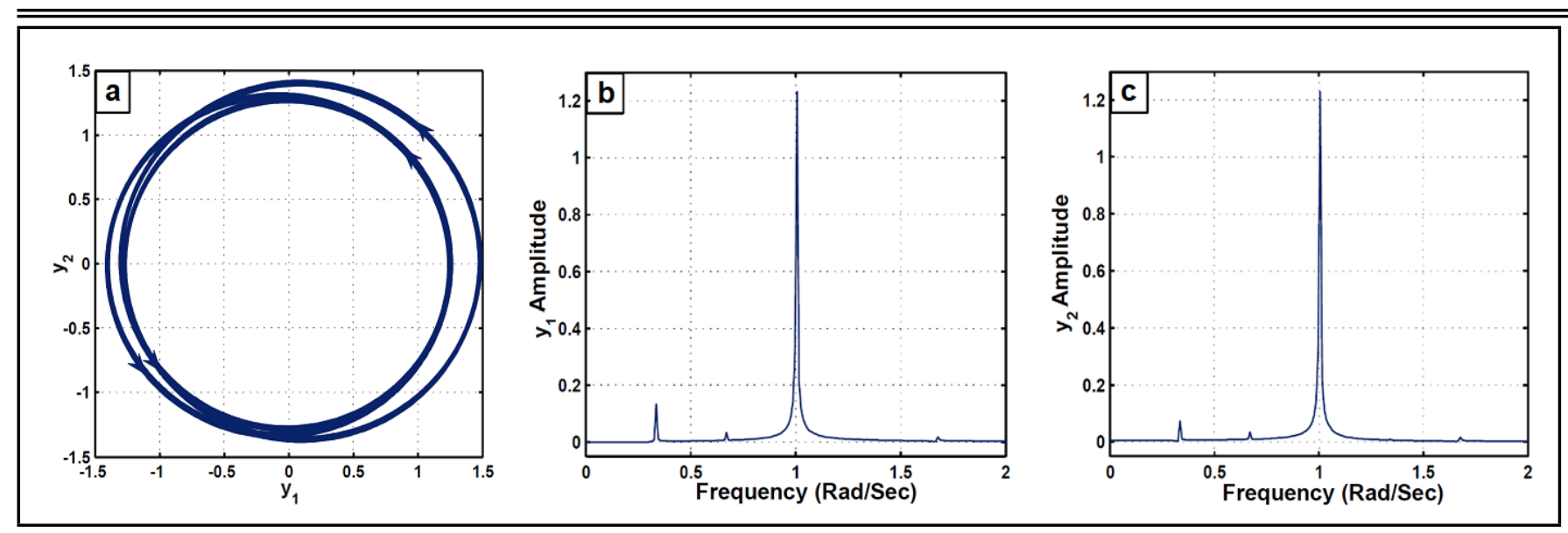

Figure 23. Simulation of point $p_{1}$ on Fig. 22. (i.e. $\alpha=0.05, \Omega=\frac{2}{3} \omega+0.02$ ): (a) Jeffcott rotor whirling orbit and (c, d) frequency-spectrum when the disc center $G$ starts rotation from the position $y_{1}(0)=y_{2}(0)=1.5$.

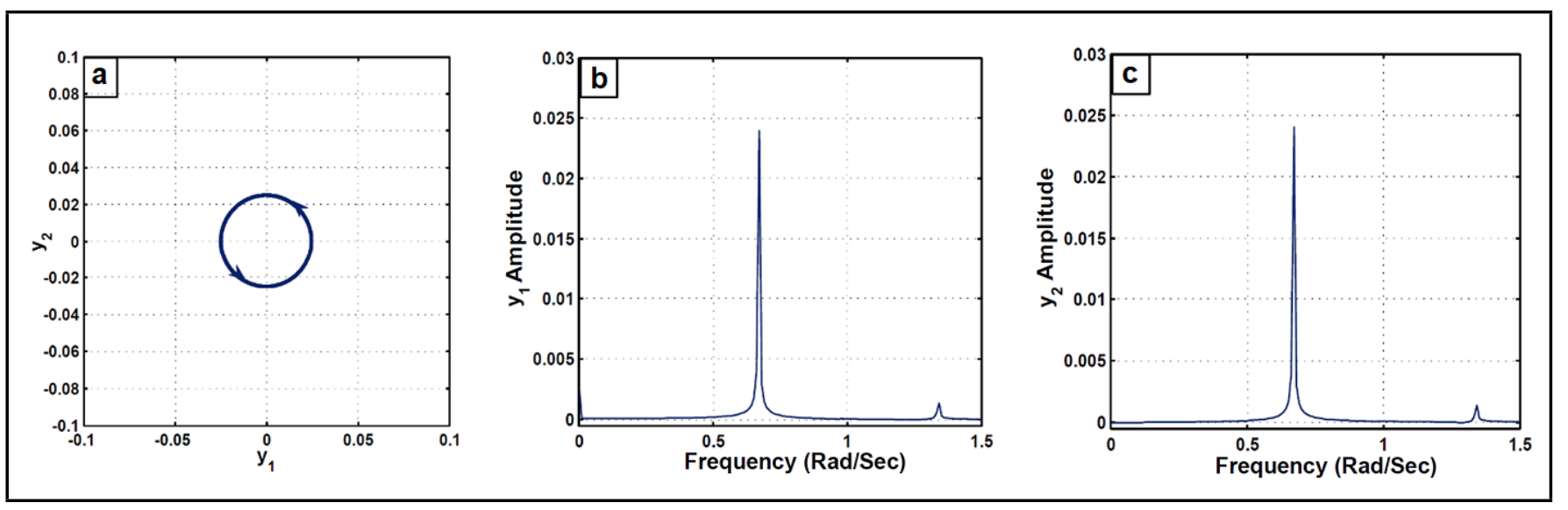

Figure 24. Simulation of point $p_{2}$ on Fig. 22. (i.e. $\alpha=0.05, \Omega=\frac{2}{3} \omega+0.02$ ): (a) Jeffcott rotor whirling orbit and (c, d) frequency-spectrum when the disc center $G$ starts rotation from the position $y_{1}(0)=y_{2}(0)=0.0$.

of Non-Linear Mechanics, 42, 959-972, (2007). https://dx.doi.org/10.1016/j.ijnonlinmec.2007.04.002

${ }^{8}$ Sinou, J. J. and Lees, A. W. A non-linear study of a cracked rotor, European Journal of Mechanics-A/Solids, 26, 152-170, (2007). https://dx.doi.org/10.1016/j.euromechsol.2006.04.002

9 Sinou, J. J. Detection of cracks in rotor based on the $2 \times$ and $3 \times$ super-harmonic frequency components and the crack-imbalance interactions, Communications in Nonlinear Science and Numerical Simulation, 13, 2024-2040, (2008). https://dx.doi.org/10.1016/j.cnsns.2007.04.008

10 Chen, C. P., Dai, L. M., and Fu, Y. M. Nonlinear response and dynamic stability of a cracked rotor, Communications in Nonlinear Science and Numerical Simulation, 12, 1023-1037, (2007). https://dx.doi.org/10.1016/j.cnsns.2005.09.004

11 Han, Q. K. and Chu, F. L. Parametric instability of a rotor bearing system with two breathing transverse cracks, European Journal of Mechanics-A/Solids, 36, 180-190, (2012). https://dx.doi.org/10.1016/j.euromechsol.2012.03.003

12 Dai, L. M. and Chen, C. P. Dynamic stability analysis of a cracked nonlinear rotor system subjected to periodic excitations in machining, Journal of Vibration and Control, 13, 537-556, (2007). https://dx.doi.org/10.1177/1077546307074242

${ }^{13}$ Lin, Y. and Chu, F. Numerical and experimental investigations of flexural vibrations of a rotor system with transverse or slant crack, Journal of Sound and Vibration, 324, 107125, (2009). https://dx.doi.org/10.1016/j.jsv.2009.01.058

14 AL-Shudeifat, M. A., Butcher, E. A., and Stern, C. R. General harmonic balance solution of a cracked rotorbearing-disc system for harmonic and sub-harmonic analysis: Analytical and experimental approach, International Journal of Engineering Science, 48, 921-935, (2010). https://dx.doi.org/10.1016/j.ijengsci.2010.05.012

15 Jun, O. S. and Gadala, M. S. Dynamic behavior analysis of cracked rotor, Journal of Sound and Vibration, 309, 210245, (2008). https://dx.doi.org/10.1016/j.jsv.2007.06.065

16 Jun, O. S. Dynamic behavior analysis of cracked rotor based on harmonic motion, Mechanical Systems and Signal Processing, 30, 186-203, (2012). https://dx.doi.org/10.1016/j.ymssp.2012.01.014

17 Ishida, Y. Cracked rotors: Industrial machine case histories and nonlinear effects shown by simple Jeffcott rotor, 
Mechanical Systems and Signal Processing, 22, 805-817, (2008). https://dx.doi.org/10.1016/j.ymssp.2007.11.005

18 Hou, L., Chen, Y., Lu, Z., and Li, Z. Bifurcation analysis for 2:1 and 3:1 super-harmonic resonances of an aircraft cracked rotor system due to maneuver load, Nonlinear Dynamics, 81, 531-547, (2015). https://dx.doi.org/10.1007/s11071-015-2009-1

19 Ishida, Y. and Inoue, T. Vibration Suppression of nonlinear rotor systems using a dynamic damper, Journal of Vibration and Control, 13 (8), 1127-1143, (2007). https://dx.doi.org/10.1177/1077546307074577

20 Yamamoto, T. and Ishida, Y. Linear and Nonlinear Rotor Dynamics, Wiley-VCH, Weinheim, Germany, (2012).

21 Nayfeh, A. Resolving controversies in the application of the method of multiple scales and the generalized method of averaging, Nonlinear Dynamics, 40, 61-102, (2005). https://dx.doi.org/10.1007/s11071-005-3937-y

22 Nayfeh, A. and Mook, D. Nonlinear Oscillations, Wiley, New York, (1995). https://dx.doi.org/10.1002/9783527617586

23 Nayfeh, A. Perturbation Methods, Wiley, New York, (1973).

24 Slotine, J.-J. E. and Li, W. Applied Nonlinear Control, Englewood Cliffs, New Jersey: Prentice Hall, (1991).

\section{APPENDIX}

Coefficients of Jacobian matrix at primary resonance case:

$$
\begin{aligned}
& \delta_{11}=\frac{1}{8 \omega}\left(-4 \mu_{1} \omega+\beta a_{20}^{2} \sin \left(2 \phi_{20}-2 \phi_{10}\right)+2 \alpha \sin \left(2 \phi_{10}\right)\right) ; \\
& \delta_{12}=\frac{1}{4 \omega}\left(\beta a_{10} a_{20} \sin \left(2 \phi_{20}-2 \phi_{10}\right)-\alpha \cos \left(\phi_{10}+\phi_{20}\right)\right) \text {; } \\
& \delta_{13}=\frac{1}{4 \omega}\left(-\beta a_{10} a_{20}^{2} \cos \left(2 \phi_{20}-2 \phi_{10}\right)+2 f \Omega^{2} \cos \left(\phi_{10}+\gamma\right)+\right. \\
& \left.2 \alpha a_{10} \cos \left(2 \phi_{10}\right)+\alpha a_{20} \sin \left(\phi_{10}+\phi_{20}\right)\right) \text {; } \\
& \delta_{14}=\frac{1}{4 \omega}\left(\beta a_{10} a_{20}^{2} \cos \left(2 \phi_{20}-2 \phi_{10}\right)+\alpha a_{20} \sin \left(\phi_{10}+\phi_{20}\right)\right) \text {; } \\
& \delta_{21}=\frac{-1}{4 \omega}\left(\beta a_{10} a_{20} \sin \left(2 \phi_{20}-2 \phi_{10}\right)+\alpha \cos \left(\phi_{10}+\phi_{20}\right)\right) \text {; } \\
& \delta_{22}=\frac{-1}{8 \omega}\left(4 \mu_{2} \omega+\beta a_{10}^{2} \sin \left(2 \phi_{20}-2 \phi_{10}\right)+2 \alpha \sin \left(2 \phi_{20}\right)\right) \text {; } \\
& \delta_{23}=\frac{1}{4 \omega}\left(\beta a_{10}^{2} a_{20} \cos \left(2 \phi_{20}-2 \phi_{10}\right)+\alpha a_{10} \sin \left(\phi_{10}+\phi_{20}\right)\right) \text {; } \\
& \delta_{24}=\frac{1}{4 \omega}\left(-\beta a_{10}^{2} a_{20} \cos \left(2 \phi_{20}-2 \phi_{10}\right)+2 f \Omega^{2} \sin \left(\phi_{20}+\gamma\right)-\right. \\
& \left.2 \alpha a_{20} \cos \left(2 \phi_{20}\right)+\alpha a_{10} \sin \left(\phi_{10}+\phi_{20}\right)\right) \text {; } \\
& \delta_{31}=\frac{-1}{4 \omega a_{10}^{2}}\left(3 \beta a_{10}^{3}+2 f \Omega^{2} \cos \left(\phi_{10}+\gamma\right)+\right. \\
& \left.\alpha a_{20} \sin \left(\phi_{10}+\phi_{20}\right)\right) \text {; } \\
& \delta_{32}=\frac{1}{4 \omega a_{10}}\left(-\beta a_{10} a_{20} \cos \left(2 \phi_{20}-2 \phi_{10}\right)-2 \beta a_{10} a_{20}+\right. \\
& \left.\alpha \sin \left(\phi_{10}+\phi_{20}\right)\right) \text {; }
\end{aligned}
$$

$$
\begin{aligned}
\delta_{33}= & \frac{1}{4 \omega a_{10}}\left(\beta a_{10} a_{20}^{2} \sin \left(2 \phi_{10}\right)-f \Omega^{2} \sin \left(\phi_{10}+\gamma\right)-\right. \\
& \left.2 \alpha a_{10} \sin \left(2 \phi_{10}\right)+\alpha a_{20} \cos \left(\phi_{10}+\phi_{20}\right)\right) ; \\
\delta_{34}= & \frac{1}{4 \omega a_{10}}\left(\beta a_{10} a_{20}^{2} \sin \left(2 \phi_{20}-2 \phi_{10}\right)+\right. \\
& \left.\alpha a_{20} \cos \left(\phi_{10}+\phi_{20}\right)\right) ; \\
\delta_{41}= & \frac{1}{4 \omega a_{20}}\left(-\beta a_{10} a_{20} \cos \left(2 \phi_{20}-2 \phi_{10}\right)-2 \beta a_{10} a_{20}+\right. \\
& \left.\alpha \sin \left(\phi_{10}+\phi_{20}\right)\right) ; \\
\delta_{42}= & \frac{-1}{4 \omega a_{20}^{2}}\left(3 \beta a_{20}^{3}+2 f \Omega^{2} \sin \left(\phi_{20}+\gamma\right)+\right. \\
& \left.\alpha a_{10} \sin \left(\phi_{10}+\phi_{20}\right)\right) ; \\
\delta_{43}= & \frac{1}{4 \omega a_{20}}\left(\beta a_{10}^{2} a_{20} \sin \left(2 \phi_{20}-2 \phi_{10}\right)+\right. \\
& \left.\alpha a_{10} \cos \left(\phi_{10}+\phi_{20}\right)\right) ; \\
\delta_{44}= & \frac{1}{4 \omega a_{20}}\left(\beta a_{10}^{2} a_{20} \sin \left(2 \phi_{20}-2 \phi_{10}\right)+2 f \Omega^{2} \cos \left(\phi_{20}+\gamma\right)+\right. \\
& \left.2 \alpha a_{20} \sin \left(2 \phi_{20}\right)+\alpha a_{10} \cos \left(\phi_{10}+\phi_{20}\right)\right) .
\end{aligned}
$$

Coefficients of Jacobian matrix at sub-harmonic resonance case:

$$
\begin{aligned}
\delta_{11}= & \frac{1}{8 \omega}\left(-4 \mu_{1} \omega-\beta a_{20}^{2} \sin \left(\phi_{10}-\phi_{20}\right)+3 \alpha \sin \left(\phi_{10}\right)\right) \\
\delta_{12}= & \frac{-1}{8 \omega}\left(2 \beta a_{10} a_{20} \sin \left(\phi_{10}-\phi_{20}\right)+\alpha \cos \left(\frac{\phi_{10}+\phi_{20}}{2}\right)\right) \\
\delta_{13}= & \frac{1}{16 \omega}\left(-2 \beta a_{10} a_{20}^{2} \cos \left(\phi_{10}-\phi_{20}\right)+6 \alpha a_{10} \cos \left(\phi_{10}\right)+\right. \\
& \left.\alpha a_{20} \sin \left(\frac{\phi_{10}+\phi_{20}}{2}\right)\right)
\end{aligned}
$$$$
\delta_{14}=\frac{1}{8 \omega}\left(\beta a_{10} a_{20}^{2} \cos \left(\phi_{10}-\phi_{20}\right)+\alpha a_{20} \sin \left(\frac{\phi_{10}+\phi_{20}}{2}\right)\right) \text {; }
$$$$
\delta_{21}=\frac{-1}{8 \omega}\left(2 \beta a_{10} a_{20} \sin \left(\phi_{20}-\phi_{10}\right)+\alpha \cos \left(\frac{\phi_{10}+\phi_{20}}{2}\right)\right) \text {; }
$$$$
\delta_{22}=\frac{1}{8 \omega}\left(-4 \mu_{2} \omega-\beta a_{10}^{2} \sin \left(\phi_{20}-\phi_{10}\right)+\alpha \sin \left(\phi_{20}\right)\right) ;
$$$$
\delta_{23}=\frac{1}{16 \omega}\left(2 \beta a_{10}^{2} a_{20} \cos \left(\phi_{20}-\phi_{10}\right)+\alpha a_{10} \sin \left(\frac{\phi_{10}+\phi_{20}}{2}\right)\right) ;
$$$$
\delta_{24}=\frac{1}{16 \omega}\left(-2 \beta a_{10}^{2} a_{20} \cos \left(\phi_{20}-\phi_{10}\right)+2 \alpha a_{20} \cos \left(\phi_{20}\right)+\right.
$$$$
\left.\alpha a_{10} \sin \left(\frac{\phi_{10}+\phi_{20}}{2}\right)\right)
$$$$
\delta_{31}=\frac{-1}{4 \omega a_{10}^{2}}\left(6 \beta a_{10}^{3}+\alpha a_{20} \sin \left(\frac{\phi_{10}+\phi_{20}}{2}\right)\right) ;
$$$$
\delta_{32}=\frac{1}{4 \omega a_{10}}\left(-2 \beta a_{10} a_{20} \cos \left(\phi_{10}-\phi_{20}\right)-4 \beta a_{10} a_{20}+\right.
$$$$
\left.\alpha \sin \left(\frac{\phi_{10}+\phi_{20}}{2}\right)\right) \text {; }
$$$$
\delta_{33}=\frac{1}{8 \omega a_{10}}\left(2 \beta a_{10} a_{20}^{2} \sin \left(\phi_{10}-\phi_{20}\right)-6 \alpha a_{10} \sin \left(\phi_{10}\right)+\right.
$$$$
\left.\alpha a_{20} \cos \left(\frac{\phi_{10}+\phi_{20}}{2}\right)\right) \text {; }
$$ 


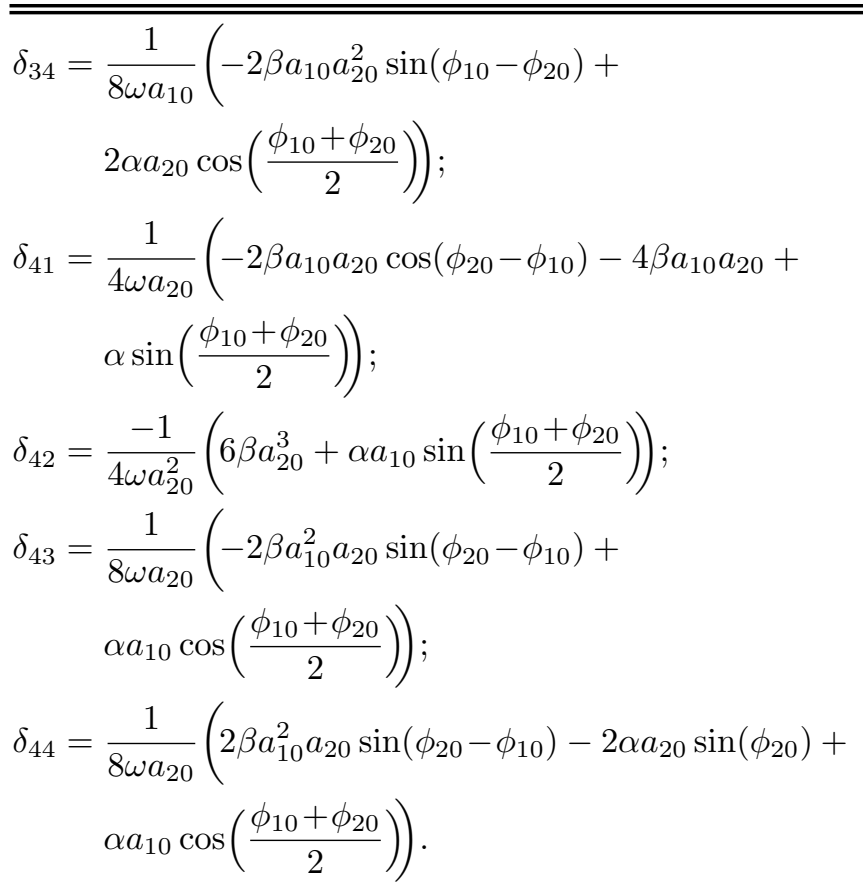

Coefficients of Jacobian matrix at the super-harmonic resonance case:

$$
\begin{aligned}
\delta_{11}= & \frac{1}{8 \omega}\left(-4 \mu_{1} \omega+\beta a_{20}^{2} \sin \left(\phi_{20}-\phi_{10}\right)+\alpha \sin \left(\phi_{10}\right)\right) ; \\
\delta_{12}= & \frac{1}{8 \omega}\left(2 \beta a_{10} a_{20} \sin \left(\phi_{20}-\phi_{10}\right)-\alpha \cos \left(\frac{\phi_{10}+\phi_{20}}{2}\right)\right) ; \\
\delta_{13}= & \frac{1}{16 \omega}\left(-2 \beta a_{10} a_{20}^{2} \cos \left(\phi_{20}-\phi_{10}\right)+2 \alpha a_{10} \cos \left(\phi_{10}\right)+\right. \\
& \left.\alpha a_{20} \sin \left(\frac{\phi_{10}+\phi_{20}}{2}\right)\right) ; \\
\delta_{14}= & \frac{1}{16 \omega}\left(2 \beta a_{10} a_{20}^{2} \cos \left(\phi_{20}-\phi_{10}\right)+\alpha a_{20} \sin \left(\frac{\phi_{10}+\phi_{20}}{2}\right)\right) ; \\
\delta_{21}= & \frac{-1}{8 \omega}\left(2 \beta a_{10} a_{20} \sin \left(\phi_{20}-\phi_{10}\right)+\alpha \cos \left(\frac{\phi_{10}+\phi_{20}}{2}\right)\right) ; \\
\delta_{22}= & \frac{-1}{8 \omega}\left(4 \mu_{2} \omega+\beta a_{10}^{2} \sin \left(\phi_{20}-\phi_{10}\right)+\alpha \sin \left(\phi_{20}\right)\right) ; \\
\delta_{23}= & \frac{1}{16 \omega}\left(2 \beta a_{10}^{2} a_{20} \cos \left(\phi_{20}-\phi_{10}\right)+\alpha a_{10} \sin \left(\frac{\phi_{10}+\phi_{20}}{2}\right)\right) ; \\
\delta_{24}= & \frac{-1}{16 \omega}\left(2 \beta a_{10}^{2} a_{20} \cos \left(\phi_{20}-\phi_{10}\right)+2 \alpha a_{20} \cos \left(\phi_{20}\right)+\right. \\
\delta_{32}= & \frac{1}{4 \omega a_{10}}\left(-2 \beta a_{10} a_{20} \cos \left(\phi_{20}-\phi_{10}\right)-4 \beta a_{10} a_{20}+\right. \\
& \left.\alpha a_{10} \sin \left(\frac{\phi_{10}+\phi_{20}}{2}\right)\right) ; \\
& \frac{-1}{4 \omega a_{10}^{2}}\left(6 \beta a_{10}^{3}+\alpha a_{20} \sin \left(\frac{\phi_{10}+\phi_{20}}{2}\right)\right) ;
\end{aligned}
$$

$$
\begin{aligned}
& \delta_{33}= \frac{1}{8 \omega a_{10}}\left(-2 \beta a_{10} a_{20}^{2} \sin \left(\phi_{20}-\phi_{10}\right)-2 \alpha a_{10} \sin \left(\phi_{10}\right)+\right. \\
&\left.\alpha a_{20} \cos \left(\frac{\phi_{10}+\phi_{20}}{2}\right)\right) ; \\
& \delta_{34}= \frac{1}{8 \omega a_{10}}\left(2 \beta a_{10} a_{20}^{2} \sin \left(\phi_{20}-\phi_{10}\right)+\right. \\
&\left.\alpha a_{20} \cos \left(\frac{\phi_{10}+\phi_{20}}{2}\right)\right) ; \\
& \delta_{41}= \frac{1}{4 \omega a_{20}}\left(-2 \beta a_{10} a_{20} \cos \left(\phi_{20}-\phi_{10}\right)-4 \beta a_{10} a_{20}+\right. \\
&\left.\alpha \sin \left(\frac{\phi_{10}+\phi_{20}}{2}\right)\right) ; \\
& \delta_{42}= \frac{-1}{4 \omega a_{20}^{2}}\left(6 \beta a_{20}^{3}+\alpha a_{10} \sin \left(\frac{\phi_{10}+\phi_{20}}{2}\right)\right) ; \\
& \delta_{43}= \frac{1}{8 \omega a_{20}}\left(-2 \beta a_{10}^{2} a_{20} \sin \left(\phi_{20}-\phi_{10}\right)+\right. \\
&\left.\alpha a_{10} \cos \left(\frac{\phi_{10}+\phi_{20}}{2}\right)\right) ;
\end{aligned}
$$$$
\delta_{44}=\frac{1}{8 \omega a_{20}}\left(2 \beta a_{10}^{2} a_{20} \sin \left(\phi_{20}-\phi_{10}\right)+2 \alpha a_{20} \sin \left(\phi_{20}\right)+\right.
$$$$
\left.\alpha a_{10} \cos \left(\frac{\phi_{10}+\phi_{20}}{2}\right)\right) \text {. }
$$

Coefficients of Eq. (26):

$$
\begin{aligned}
\Delta_{1}= & -\left(\delta_{11}+\delta_{22}+\delta_{33}+\delta_{44}\right) \\
\Delta_{2}= & \delta_{11} \delta_{44}+\delta_{11} \delta_{33}+\delta_{11} \delta_{22}+\delta_{22} \delta_{44}+\delta_{22} \delta_{33}+ \\
& \delta_{33} \delta_{44}-\delta_{21} \delta_{12}-\delta_{31} \delta_{13}-\delta_{41} \delta_{14}-\delta_{34} \delta_{43}- \\
& \delta_{32} \delta_{23}-\delta_{42} \delta_{24} ; \\
\Delta_{3}= & \delta_{11} \delta_{32} \delta_{22}-\delta_{31} \delta_{12} \delta_{23}-\delta_{11} \delta_{22} \delta_{44}-\delta_{42} \delta_{23} \delta_{34}+ \\
& \delta_{31} \delta_{22} \delta_{13}+\delta_{32} \delta_{23} \delta_{44}-\delta_{11} \delta_{22} \delta_{33}-\delta_{32} \delta_{43} \delta_{24}- \\
& \delta_{21} \delta_{32} \delta_{13}+\delta_{11} \delta_{42} \delta_{24}+\delta_{31} \delta_{13} \delta_{44}+\delta_{21} \delta_{12} \delta_{44}- \\
& \delta_{21} \delta_{42} \delta_{14}+\delta_{42} \delta_{24} \delta_{33}+\delta_{21} \delta_{12} \delta_{33}-\delta_{11} \delta_{33} \delta_{44}- \\
& \delta_{22} \delta_{33} \delta_{44}-\delta_{41} \delta_{12} \delta_{24}-\delta_{31} \delta_{43} \delta_{14}+\delta_{41} \delta_{22} \delta_{14}+ \\
& \delta_{22} \delta_{34} \delta_{44}+\delta_{41} \delta_{14} \delta_{33}+\delta_{11} \delta_{34} \delta_{43}-\delta_{41} \delta_{13} \delta_{34} \\
\Delta_{4}= & -\delta_{41} \delta_{12} \delta_{23} \delta_{34}-\delta_{21} \delta_{12} \delta_{33} \delta_{44}+\delta_{11} \delta_{22} \delta_{33} \delta_{44}- \\
& \delta_{11} \delta_{42} \delta_{24} \delta_{33}+\delta_{21} \delta_{32} \delta_{13} \delta_{44}+\delta_{11} \delta_{32} \delta_{43} \delta_{24}- \\
& \delta_{41} \delta_{32} \delta_{13} \delta_{24}+\delta_{41} \delta_{32} \delta_{23} \delta_{14}-\delta_{11} \delta_{22} \delta_{34} \delta_{43}+ \\
& \delta_{21} \delta_{12} \delta_{34} \delta_{43}-\delta_{31} \delta_{12} \delta_{43} \delta_{24}-\delta_{21} \delta_{42} \delta_{13} \delta_{34}- \\
& \delta_{21} \delta_{32} \delta_{43} \delta_{14}+\delta_{31} \delta_{12} \delta_{14} \delta_{44}+\delta_{31} \delta_{42} \delta_{13} \delta_{24}- \\
& \delta_{31} \delta_{42} \delta_{23} \delta_{14}+\delta_{21} \delta_{42} \delta_{14} \delta_{33}+\delta_{11} \delta_{42} \delta_{23} \delta_{34}- \\
& \delta_{11} \delta_{32} \delta_{23} \delta_{44}+\delta_{31} \delta_{22} \delta_{43} \delta_{14}-\delta_{31} \delta_{22} \delta_{13} \delta_{44}+ \\
& \delta_{41} \delta_{12} \delta_{24} \delta_{33}-\delta_{41} \delta_{22} \delta_{14} \delta_{33}+\delta_{41} \delta_{22} \delta_{13} \delta_{34} .
\end{aligned}
$$

